\title{
Guía de endoscopia en enfermedad inflamatoria intestinal
}

\author{
Jony Cerna-Cardona ${ }^{1 *}$, Mario C. Peláez-Luna ${ }^{2,3,4}$, Gretel B. Casillas-Guzmán ${ }^{5}$, \\ Xochiquetzal Sánchez-Chávez ${ }^{6}$, Azucena I. Casanova-Lara7, Hector Espino-Cortés², \\ Andrés S. Hernández-Ángeles ${ }^{8}$, Eumir I. Juárez-Valdés ${ }^{9}$, María E. López-Acosta ${ }^{10}$, \\ Jesús G. López-Gómez ${ }^{11}$, Clara L. Martínez-Garcia'12, Alejandra Noble-Lugo ${ }^{13}$, \\ Ángel A. Reyes-Dorantes ${ }^{14}$, Moisés Salamanca ${ }^{15}$, Omar E. Trujillo-Benavides ${ }^{16}$, \\ Ylse Gutiérrez-Grobe ${ }^{17,18}$, Luis Eduardo Zamora-Nava ${ }^{19}$, Patricio C. Acosta Rodríguez-Bueno ${ }^{20}$, \\ Miguel Á. Herrera-Servin ${ }^{17}$ y Justo A. Fernández-Rivero ${ }^{18}$
}

${ }^{1}$ Unidad de Endoscopia, Hospital Juárez de México, Ciudad de México; ${ }^{2}$ División de Investigación, Facultad de Medicina, UNAM, Ciudad de México; ${ }^{3}$ Departamento de Gastroenterología, Instituto Nacional de Ciencias Médicas y Nutrición Salvador Zubirán, Ciudad de México; ${ }^{4}$ Unidad de Endoscopia avanzada, Centro Médico ABC, Ciudad de México; 5 Unidad de Endoscopia, Centro Médico Nacional Siglo XXI, IMSS, Ciudad de México; ${ }^{6}$ Servicio de Gastroenterología y Endoscopia, Hospital San Ángel Inn Patriotrismo, Ciudad de México; ${ }^{7}$ Servicio de Endoscopia, Hospital General Regional N. ${ }^{\circ} 17$, IMSS, Cancún, Q. R.; ${ }^{8}$ Unidad de Endoscopia, Centro de Especialidades Médicas Ángeles Tula, CEMAN, Tula, Hgo.; ${ }^{9}$ Departamento de Gastroenterología, Hospital Juárez de México, Ciudad de México; ${ }^{10}$ Unidad de Gastroenterología y Fisiología Digestiva, Hospital Ángeles Lomas, Ciudad de México; ${ }^{11}$ Clínica de Enfermedad Inflamatoria intestinal, Hospital 20 de Noviembre, ISSSTE, Ciudad de México; ${ }^{12}$ Unidad Endoscopia Digestiva, Hospital San Ángel Inn Universidad, Ciudad de México; ${ }^{13}$ Servicio de Gastroenterologíal Departamento de Enseñanza e Investigación, Hospital Español de México, Ciudad de México; ${ }^{14}$ Servicio de Gastroenterología y Endoscopia, Hospital San Ángel Inn Chapultepec, Ciudad de México; ${ }^{15}$ Servicio de Patología, Hospital 20 de Noviembre, ISSSTE, Ciudad de México; ${ }^{16}$ Servicio de Gastroenterología, Hospital General de Zona N. ${ }^{\circ} 42$, IMSS, Puerto Vallarta, Jal.; ${ }^{17}$ Departamento de Gastroenterología, Hospital General de México Dr. Eduardo Liceaga, Ciudad de México; ${ }^{18}$ Unidad de Endoscopia, Clínica de Enfermedades Digestivas y Obesidad, Fundación Clínica, Médica Sur, Ciudad de México; ${ }^{19}$ Unidad de Endoscopia, Instituto Nacional de Ciencias Médicas y Nutrición Salvador Zubirán, Ciudad de México; ${ }^{20}$ Servicio de Pediatría Mixta, Hospital Infantil de México Dr. Federico Gómez, Ciudad de México. México

\section{Resumen}

En México la incidencia de la enfermedad inflamatoria intestinal ha incrementado. Esto hace que el especialista tenga mayor conocimiento de las herramientas diagnósticas disponibles, terapias farmacológicas y seguimiento de la enfermedad, lo que impacta en la calidad de vida del paciente, reducción de la morbilidad y mortalidad. La Asociación Mexicana de Endoscopia Gastrointestinal convocó de manera virtual a especialistas en gastroenterología, endoscopia y enfermedad inflamatoria intestinal de instituciones públicas y privadas del país, con la finalidad de evaluar de manera crítica la evidencia científica disponible y elaborar las guías clínicas de endoscopia en enfermedad inflamatoria intestinal.

Palabras clave: Endoscopia. Colitis ulcerosa. Enfermedad de Crohn.

\section{Endoscopy guideline in inflammatory bowel disease}

\author{
Abstract \\ The incidence of inflammatory bowel disease in Mexico has increased. This makes the specialist to have a better knowledge \\ of the available diagnostic tools, pharmacological therapies and follow-up of the disease, which has an impact on the qual- \\ Correspondencia: \\ *Jony Cerna-Cardona \\ E-mail: jonycerna_80@ hotmail.com \\ DOI: 10.24875/END.M21000329 \\ Fecha de recepción: 04-02-2021 \\ Fecha de aceptación: 09-02-2021 \\ Disponible en internet: 12-03-2021 \\ Endoscopia. 2021;33(1):22-44 \\ www.endoscopia-ameg.com
}


ity of life of the patient and a reduction in morbidity and mortality. The Asociación Mexicana de Endoscopia Gastrointestinal, convened in a virtual way specialists in gastroenterology, endoscopy and inflammatory bowel disease from public and private institutions in the country, in order to critically evaluate the available scientific evidence and develop the clinical guidelines for endoscopy in inflammatory bowel disease.

Key words: Endoscopy. Ulcerative colitis. Crohn's disease.

\section{Introducción}

El término enfermedad inflamatoria intestinal (Ell) agrupa a condiciones inflamatorias crónicas recurrentes del tubo digestivo caracterizadas por una respuesta inmunológica alterada, siendo las más comunes la enfermedad de Crohn (EC) y la colitis ulcerosa (CU). Estas tienen un impacto negativo en la calidad de vida y comportan un riesgo elevado para el desarrollo de cáncer colorrectal (CCR).

El diagnóstico requiere una adecuada historia clínica, exploración física, estudios de laboratorio, imagen, endoscopia e histología. La endoscopia constituye uno de los pilares fundamentales para el diagnóstico y seguimiento de la enfermedad y para el escrutinio de CCR asociado a estas patologías y uso de técnicas terapéuticas para resolución de complicaciones. Las herramientas endoscópicas útiles en la evaluación y manejo de la Ell incluyen la cápsula endoscópica (CE), enteroscopia y cromoendoscopia, entre otras. La indicación de cada una de las técnicas endoscópicas es variable, pues depende de las características de cada paciente, disponibilidad de equipo y experiencia del endoscopista. Debido a esto, la Asociación Mexicana de Endoscopia Gastrointestinal (AMEG) y Colegio de Profesionistas AC congregó de manera virtual el 18 y 25 de junio del 2020 a especialistas en Gastroenterología, Endoscopia y Enfermedad Inflamatoria Intestinal de instituciones públicas y privadas del país, para revisar la evidencia actual y elaborar las Guías Clínicas de Endoscopia en Enfermedad Inflamatoria Intestinal.

\section{Metodología}

Se utilizó el método Delphi. Se crearon cuatro mesas de trabajo y a cada una se le asignó un tema específico que representa cada una de las secciones de la presente guía. Seis de los participantes realizaron una búsqueda en la base de datos PubMed de artículos publicados a manera de resumen o artículo completo en los idiomas inglés y español. Se dio preferencia a revisiones sistemáticas, metaanálisis, guías de práctica clínica, consensos, ensayos clínicos controlados y cohortes, aunque no se limitó a este tipo de manuscritos.
Una vez concluida la búsqueda se elaboraron enunciados relacionados con el tema de cada mesa, mismos que fueron evaluados y votados por el comité organizador y fueron enviados a cada coordinador de mesa junto con la bibliografía seleccionada. Cada coordinador asignó aleatoriamente uno o más enunciados a cada participante, quien calificó el grado de la evidencia que los apoya.

Para calificar la evidencia y seleccionar los artículos más relevantes sin importar si los resultados eran positivos o negativos se utilizó el sistema GRADE (Grading of Recommendations, Assessment, Development and Evaluation) modificado.

Durante la reunión virtual, los enunciados fueron presentados por los coordinadores a los miembros de cada mesa, donde se revisó el contenido y redacción de estos efectuando modificaciones en conjunto, además de discutir la pertinencia o necesidad de eliminar o agregar otros enunciados.

Se votó «en acuerdo» o «en desacuerdo» para cada enunciado. Cuando el acuerdo era mayor al $75 \%$ se concluyó que el enunciado permanecería sin modificaciones, y cuando el acuerdo era menor al $75 \%$ se realizaron modificaciones en contenido y redacción, sometiéndose nuevamente a votación, y así sucesivamente hasta llegar a un acuerdo mayor al $75 \%$. Aquellos enunciados con desacuerdo mayor al $75 \%$, repetidos o redundantes, fueron eliminados del proceso.

\section{Definiciones}

\section{Enfermedad inflamatoria intestinal}

La Ell es un grupo de condiciones inflamatorias crónicas recurrentes del tubo digestivo caracterizado por una respuesta inmunológica alterada ${ }^{1,2}$.

\section{Colitis ulcerosa}

La CU es un proceso inflamatorio crónico confinado al colon, que se caracteriza clínicamente por diarrea con moco y sangre, dolor abdominal y pérdida de peso. La inflamación del colon es superficial (mucosa) y 
continua, con inicio en el recto y de extensión proximal. Histológicamente se puede observar edema, congestión de la mucosa, infiltración de linfocitos, células plasmáticas, polimorfonucleares y granulocitos, alteración de las células caliciformes, abscesos de criptas, distorsión de la arquitectura de las criptas y ulceración ${ }^{1-3}$.

\section{Enfermedad de Crohn}

La EC es un proceso inflamatorio crónico que puede afectar a cualquier parte del tubo digestivo (desde la boca hasta el ano), manifestado por dolor abdominal, diarrea crónica y pérdida de peso. El proceso inflamatorio es focal, segmentario y transmural, a menudo complicado con fisuras, fístulas, abscesos y obstrucción intestinal. Endoscópicamente se caracteriza por úlceras aftosas e histológicamente por agregados linfoides, infiltrado inflamatorio crónico y granulomas epitelioides (menos de un 30\%) ${ }^{1-3}$.

\section{Colitis no clasificada}

Es una entidad clínico-patológica caracterizada por un proceso inflamatorio crónico intestinal que no puede llegar a clasificarse claramente como EC ni como CU. Representa el 7-10\% de la Ell ${ }^{1-3}$.

\section{Colitis indeterminada}

Hace referencia a aquellas colitis intervenidas quirúrgicamente (colectomía) en las que sea imposible establecer definitivamente si se trata de $\mathrm{EC} \circ \mathrm{CU}^{3}$.

\section{Actividad de la enfermedad}

Conjunto de parámetros clínicos, de laboratorio, endoscópicos y de gabinete que permiten establecer la gravedad de la enfermedad y normar la conducta terapéutica ${ }^{3}$.

\section{Inducción a la remisión}

Fase inicial del tratamiento farmacológico que permite lograr objetivos de reducción en los índices de actividad en la Ell. Debe ser alcanzada en el tiempo establecido y modifica la historia natural de la enfermedad ${ }^{4}$.

\section{Mantenimiento de la remisión}

Intervenciones utilizadas que logran la persistencia de la respuesta lograda en la fase de inducción a largo plazo. Se caracteriza por mejoría de los síntomas y normalización de los marcadores sanguíneos ${ }^{4,5}$.

\section{Remisión clínica}

Resolución completa de los síntomas y signos clínicos de la Ell.

\section{Remisión endoscópica}

Se define como la cicatrización de la mucosa caracterizada por un aspecto normal de esta y ausencia de ulceraciones en el seguimiento endoscópico.

\section{Remisión histológica}

Resolución del infiltrado inflamatorio y de la distorsión de la arquitectura de las criptas ${ }^{4,6,7}$.

\section{Epidemiología de la enfermedad inflamatoria intestinal}

\section{LA PREVALENCIA E INCIDENCIA DE LA EII HA INCREMENTADO EN MÉXICO. LA COLITIS ULCEROSA (CU) ES MÁS FRECUENTE QUE LA ENFERMEDAD DE CROHN (EC)}

GRADE IB (recomendación fuerte, nivel de la evidencia moderado).

La epidemiología de la Ell ha cambiado a partir de la segunda mitad del siglo XX, cuando la incidencia y prevalencia tanto de la CU como de la EC aumentaron notoriamente en los países occidentales. En estos países la incidencia y la prevalencia tienden a ser iguales para EC y CU ${ }^{8-10}$.

En los países con economías emergentes la incidencia y prevalencia de la Ell están aumentando. La mayoría de los estudios no muestran diferencia en cuanto a sexos en la Ell. La mayor incidencia de Ell se encuentra en el intervalo de 20 a 30 años, en la CU se ha observado una segunda elevación en la frecuencia entre los 60 y 79 años ${ }^{9}$.

Los estudios epidemiológicos de Ell en América Latina son heterogéneos. Una revisión sistemática de 25 estudios latinoamericanos publicados entre 2002 y 2015 informa una incidencia para EC de 0.24 a 3.50 y para CU de 0.74 a 6.76 por 100,000 habitantes. La prevalencia para EC fue de 0.24 a 14.90 y para CU 0.99 a 44.30 por 100,000 habitantes ${ }^{11}$. 
En otra revisión sistemática de 61 estudios entre 1999 y 2018 se observó aumento en la incidencia de EC en Brasil de 0.08 en 1988 a 5.5 por 100 mil habitantes en 2015, que igualó la proporción con $\mathrm{CU}^{12}$.

En México, el promedio anual de casos nuevos de CU aumentó de 28.8 en los años 1987-1996 a 76.1 en los años 1997-2006, por lo que la incidencia anual promedio aumentó 2.6 veces $^{13}$. En otro estudio de $\mathrm{CU}$ que incluyó 107 pacientes con $\mathrm{CU}$ atendidos entre 2004 y 2008, el número de casos nuevos por año aumentó de 17 a 29, es decir incrementó 1.7 veces ${ }^{14}$.

Yamamoto, et al. revisaron la epidemiologia de la Ell en el Sistema Nacional de Salud en el año 2015. Se registraron 9,953 casos de EC y 33,060 de CU; 1,097 pacientes con EC y 5,345 con CU requirieron hospitalización. Se registraron 68 muertes en el grupo de pacientes con EC y 453 en los pacientes con CU, para una tasa de mortalidad estandarizada directa de 1.26 para EC y 2.53 para CU. En todos los casos hubo un predominio leve del sexo femenino ${ }^{15}$.

Una cohorte en la que participaron 42 centros de todos los Estados del país, con un seguimiento entre los años 2000 y 2017, incluyó 2,645 pacientes con Ell: CU $2,073(78.3 \%)$, EC 501 (18.9\%) y $72(2.7 \%)$ con colitis no clasificada. Entre los años 2000 y 2015 la incidencia de Ell aumentó de 0.05 a 0.21 por 100,000 personas/ año, la de CU de 0.04 a 0.16 y la de EC de 0.01 a 0.04 (Fig. 1). En este mismo periodo se registró un incremento en la prevalencia de Ell de 0.30 a 1.83 casos por 100,000 habitantes. La CU aumentó de 0.23 a 1.45 y en la EC el aumento fue de 0.07 a 0.34 (Fig. 2) ${ }^{16}$.

\section{Se recomienda utilizar la escala de Truelove y Witts y el índice de la Clínica Mayo parcial para evaluar la actividad clínica de la colitis ulcerosa}

GRADE 1B (recomendación fuerte, calidad de la evidencia moderada).

Existen diferentes instrumentos validados para evaluar la actividad clínica en pacientes con CU. Actualmente se investiga la importancia de la evaluación global de la enfermedad con criterios clínicos, biomarcadores, hallazgos endoscópicos, histológicos y calidad de vida, sin embargo, aún no existe el instrumento ideal, validado y que tome en cuenta todos los parámetros anteriores.

El índice de Truelove y Witts (TyW) (Tabla 1) es útil para identificar pacientes con actividad grave de la enfermedad, definir ingreso hospitalario y uso de esteroides intravenosos ${ }^{18}$. Los pacientes con CU grave, definida por

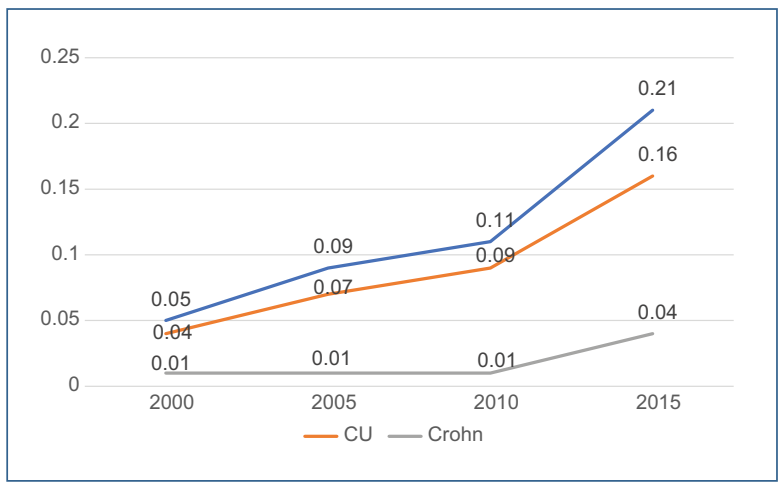

Figura 1. Incidencia de enfermedad inflamatoria intestinal (EII) en México en el siglo XXI (por 100,000 personas/año) (adaptada de Yamamoto-Furusho, et al., 202015).

CU: colitis ulcerosa.

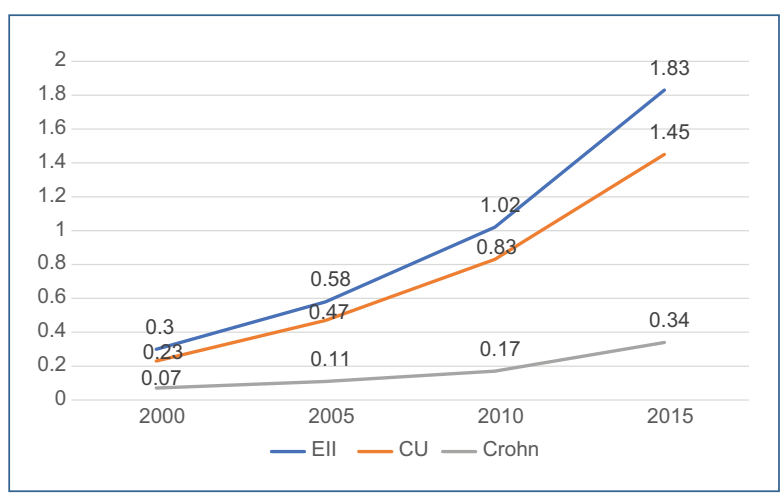

Figura 2. Prevalencia de enfermedad inflamatoria intestinal (EII) en México en el siglo XXI (por 100,000 personas/año) (adaptada de Yamamoto-Furusho, et al., 202015). CU: colitis ulcerosa.

un puntaje en la escala TyW $>22$, tienen mayor probabilidad de necesitar una colectomía a corto plazo $^{19}$.

El índice de la Clínica Mayo parcial (Tabla 2) es la escala más utilizada en ensayos clínicos y ha demostrado diferenciar adecuadamente entre enfermedad activa 0 enfermedad en remisión con un puntaje $<1$, además, ha demostrado una buena correlación con la actividad clínica y bioquímica de la enfermedad ${ }^{20}$. El índice de la Clínica Mayo completo es el estándar para evaluar la actividad de la enfermedad en el diseño de estudios que incluyen evaluación endoscópica; este índice se calcula con el número de evacuaciones, sangrado rectal, evaluación general del médico y hallazgos endoscópicos con un puntaje de 0-12 y se ha demostrado la buena correlación con la evaluación de la enfermedad en la respuesta a tratamiento ${ }^{21-23}$. 
Tabla 1. Índice cuantitativo de Truelove y Witts modificado*

\begin{tabular}{|c|c|c|c|}
\hline $\begin{array}{l}\text { Criterio clínico o } \\
\text { laboratorial }\end{array}$ & 1 & 2 & 3 \\
\hline Evacuaciones & $<4$ & $4-6$ & $>6$ \\
\hline $\begin{array}{l}\text { Sangre en las } \\
\text { evacuaciones }\end{array}$ & - & ++ & ++++ \\
\hline $\begin{array}{l}\text { Hemoglobina } \\
\text { (hombre/mujer) }\end{array}$ & $>14 />12$ & $10-14 / 10-12$ & $<10 /<10$ \\
\hline Albúmina & $>33$ & $30-32$ & $<30$ \\
\hline Temperatura ${ }^{\circ} \mathrm{C}$ & $<37$ & $37-38$ & $>38$ \\
\hline Frecuencia cardiaca & $<80$ & $80-100$ & $>100$ \\
\hline $\begin{array}{l}\text { Velocidad de } \\
\text { sedimentación } \\
\text { globular }\end{array}$ & $<15$ & $15-30$ & $>30$ \\
\hline Leucocitos $(x 1,000)$ & $<10$ & $10-13$ & $>13$ \\
\hline Potasio (mEq/l) & $>3.8$ & $3-3.8$ & $<3.8$ \\
\hline
\end{tabular}

Uno de los objetivos actuales en el manejo de la Ell es poder evaluar el éxito del tratamiento y su curso clínico de forma sencilla y veraz.

Un estudio que evaluó la variabilidad interobservador entre el índice simple de actividad clínica de colitis (criterios clínicos), el índice de la Clínica Mayo (criterios clínicos y endoscópicos) y el índice Seo (criterios clínicos y biomarcadores) encontró que las escalas con criterios endoscópicos presentan mayor variabilidad interobservador; además, el índice de la Clínica Mayo se asocia a mejor evaluación clínica en pacientes ambulatorios, lo que se atribuye a la participación del médico en la evaluación general ${ }^{17}$.

En el 2018 se validó el Nuevo Índice Integral en CU en población mexicana. Este índice considera criterios clínicos, bioquímicos, endoscópicos e histológicos y tiene buena correlación con las escalas de TyW y el índice de la Clínica Mayo, por lo que podría ser útil para la toma de decisiones y optimizar el tratamiento de la enfermedad, sin embargo, se requieren más estudios para demostrar su utilidad ${ }^{24}$.

\section{Se recomienda utilizar el índice de Harvey Bradshaw para evaluar la actividad clínica de la enfermedad de Crohn}

GRADE $1 B$ (recomendación fuerte, calidad de la evidencia moderada).
El índice de actividad de la EC (CDAl, Crohn's disease activity index) se ha utilizado para evaluar la respuesta de la enfermedad al tratamiento; un CDAI < 150 puntos indica remisión. Diversos ensayos clínicos han demostrado buena correlación con la actividad, sin embargo, los criterios de evaluación son extensos y el cálculo es complejo, además, no está validado en pacientes posquirúrgicos y no es aplicable a pacientes con estomas, por lo que su uso en la práctica clínica es limitado22,25.

El índice de Harvey-Bradshaw, que incluye solo parámetros clínicos (Tabla 3 ), permite una evaluación completa y sencilla de calcular ${ }^{26}$. Diversos estudios han mostrado adecuada correlación entre este y el índice de actividad de la EC. Un cambio de 3 puntos en el índice de Harvey-Bradshaw es igual a un cambio de 100 puntos en CDAl, lo que indica buena respuesta a tratamiento. Un puntaje menor de 4 y CDAI $<150$ indican que la enfermedad está en remisión clínica ${ }^{27}$.

La complejidad de la EC hace difícil la creación del instrumento ideal que evalúe todos los aspectos. De forma complementaria, existen otras escalas que evalúan la enfermedad después de haber recibido tratamiento quirúrgico o presencia de estoma. Las nuevas herramientas propuestas para evaluar de manera global la enfermedad toman en cuenta la evolución de la enfermedad y la calidad de vida de acuerdo con los desenlaces reportados por el paciente ${ }^{28}$.

\section{En pacientes con sospecha de enfermedad inflamatoria intestinal se recomienda realizar reactantes de fase aguda, biometría hemática, pruebas de función hepática y estudio de las heces}

GRADE $1 B$ (recomendación fuerte, nivel de evidencia moderada).

Existen diversos exámenes serológicos y fecales que tienen la ventaja de no ser invasivos, contar con alta disponibilidad y costo razonable, y que son útiles en pacientes con sospecha de Ell. Se suelen utilizar durante el abordaje diagnóstico o el seguimiento de los pacientes (con el fin de monitorizar la actividad inflamatoria, respuesta terapéutica, detectar deficiencias nutricionales y/o complicaciones); también son importantes para diagnosticar enfermedades asociadas 0 manifestaciones extraintestinales ${ }^{29-31}$.

La proteína $C$ reactiva (PCR) es inespecífica para Ell; sin embargo, refleja objetivamente la severidad de la actividad inflamatoria y permite predecir el curso de la enfermedad, monitorizar la evolución de los pacientes y su respuesta al tratamiento, con la ventaja de ser una prueba 
Tabla 2. Índice de la Clínica Mayo parcial*

\begin{tabular}{|l|l|}
\hline Criterio clínico & Valor en puntaje \\
\hline Número de evacuaciones & $\begin{array}{l}\text { 0: normal para ese paciente } \\
\text { 1: } 1-2 \text { evacuaciones por día más que lo normal para ese paciente } \\
\text { 2: } 3-4 \text { evacuaciones por día más que lo normal para ese paciente } \\
\text { 3: } \geq 5 \text { deposiciones más que lo normal para ese paciente }\end{array}$ \\
\hline Sangrado rectal & $\begin{array}{l}\text { 1: negativo } \\
\text { 2: sangre franca en más de la mitad de las deposiciones }\end{array}$ \\
\hline Valoración global del médico & 3: normal \\
\hline & 1: leve \\
\hline
\end{tabular}

*Remisión < 1, leve 2-4, moderado 5-6, severo 7-9.

Adaptada de Walsh, et al., $2014^{17}$.

Tabla 3. Índice de Harvey Bradshaw para la enfermedad de Crohn

\begin{tabular}{|l|l|l|l|l|l|}
\hline & $\mathbf{0}$ & $\mathbf{1}$ punto & $\mathbf{2}$ puntos & $\mathbf{3}$ puntos & $\mathbf{4}$ puntos \\
\hline Bienestar general & Muy bien & Ligero decaimiento & Malo & Muy malo & Pésimo \\
\hline Dolor abdominal & No & Leve & Moderado & Grave \\
\hline Masa abdominal & No & Dudosa & Definida & Definida y dolorosa \\
\hline N. ${ }^{0}$ de deposiciones líquidas al día & Sumar las deposiciones del día previo & & $\begin{array}{l}\text { Fisura anal } \\
\text { Nueva fístula } \\
\text { Cobsceso } \\
\text { Complicaciones: } 1 \text { punto por cada una }\end{array}$ \\
\hline & $\begin{array}{l}\text { Artralgias } \\
\text { Uveítis } \\
\text { Eritema nodoso } \\
\text { Úlceras aftosas }\end{array}$ & Piodenoso \\
\hline
\end{tabular}

Adaptada de Harvey, et al., $1980^{26}$.

económica. La velocidad de sedimentación lobular es un marcador inespecífico, que no correlaciona con la progresión de la Ell, por lo que su uso se ha reducido ${ }^{32}$.

En el caso de la EC, la PCR se correlaciona con la localización y gravedad de la inflamación ${ }^{32}$. Un estudio de Kiss, et al. ${ }^{33}$ encontró que los niveles de PCR ultrasensible al momento del diagnóstico de EC se correlacionan con la localización colónica o ileocolónica de la enfermedad $(p=0.002)$, un fenotipo no inflamatorio $(p=0.058)$ y mayor requerimiento de inmunosupresores y terapia biológica ( $p<0.001$ y $p=0.024$ respectivamente).

La biometría hemática completa y las pruebas de funcionamiento hepático tienen un papel relevante para descartar complicaciones de la Ell o de su tratamiento ${ }^{32}$ como son infecciones asociadas, deficiencias nutricionales (vitamina B12, ácido fólico, hierro), anemia, colangitis esclerosante primaria (CEP), hepatopatía inducida por fármacos o la presencia de esteatosis hepática. Adicionalmente, la determinación de albúmina, prealbúmina, ferritina y transferrina séricas mejoran la evaluación del estado nutricional en estos pacientes ${ }^{32}$.

Ante la sospecha diagnóstica de Ell, los estudios de materia fecal son parte importante del abordaje diagnóstico. Ayudan a descartar procesos infecciosos concomitantes como causa de recaídas, identifican casos con actividad persistente 0 con pobre respuesta al tratamiento. Se recomienda realizar examen coproparasitoscópico, coprocultivo y toxinas A y B para Clostridioides difficile ${ }^{29}$. También se puede considerar la detección de otras infecciones (como citomegalovirus [CMV]) por medio de determinación de antígenos o mediante detección de ácidos nucleicos por reacción en cadena de la polimerasa ${ }^{32}$.

Por otra parte, la determinación de proteínas derivadas de neutrófilos, como la calprotectina fecal (CF) y la lactoferrina (LF), son biomarcadores de inflamación intestinal ${ }^{30,32}$. 
La CF es un potente factor quimiotáctico de neutrófilos y se eleva en diversas alteraciones intestinales. Su determinación cuantitativa permite diferenciar entre trastornos funcionales y Ell, siendo particularmente útil en $\mathrm{CU}^{30}$. Tiene buena correlación con la actividad endoscópica de Ell, por lo que sirve también para valorar la actividad de esta, predecir recaídas y monitorizar la respuesta terapéutica de forma no invasiva, no sustituyendo a la colonoscopia para el escrutinio de CCR o de evaluación en la actividad grave ${ }^{32}$. Un metaanálisis de von Roon, et al. ${ }^{34}$ demostró que la CF tiene una sensibilidad (S) de 0.95 (intervalo de confianza del 95\% [IC 95\%]: 0.93-0.97), especificidad (E) de 0.91 (IC 95\%: 0.86-0.91) y área bajo la curva de 0.95 para el diagnóstico de Ell, utilizando un punto de corte de $100 \mu \mathrm{g} / \mathrm{g}$.

Los diversos biomarcadores varían dependiendo del tipo específico de Ell, la edad del paciente y la localización anatómica de la enfermedad. Mosli, et al. ${ }^{35}$ Ilevaron a cabo una revisión sistemática y metaanálisis para evaluar la precisión diagnóstica de la PCR, la CF y la LF en la determinación de actividad endoscópica de la Ell. La $S$ de estas pruebas fue de 0.49 (IC 95\%: 0.34-0.64), 0.92 (IC 95\%: 0.72-0.96) y 0.88 (IC 95\%: $0.84-0.90$ ), mientras que su $E$ fue de 0.73 (IC $95 \%$ : 0.66-0.79), 0.82 (IC 95\%: 0.73-0.88) y 0.79 (IC 95\%: $0.62-0.89)$, respectivamente. La CF resultó ser la prueba más sensible tanto en el caso de la CU como en la $\mathrm{EC}$, con una $\mathrm{S}$ mayor para CU.

En el caso de pacientes pediátricos con Ell, Holtman, et al. ${ }^{36}$ realizaron un metaanálisis para determinar la $S$ y la $E$ de diversos biomarcadores no invasivos, encontrando que la PCR y los niveles de albúmina sérica tienen el mejor rendimiento diagnóstico para apoyar el diagnóstico de Ell. La PCR mostró una S de 0.63 (IC 95\%: 0.51-0.73) y E de 0.88 (IC 95\%: 0.80-0.93), mientras que la albúmina tuvo S de 0.48 (IC 95\%: 0.31-0.66) y E de 0.94 (IC 95\%: 0.86-0.98). Por su parte, la CF mostró ser particularmente útil para descartar Ell con una S de 0.99 (IC 95\%: 0.921.00) y E de 0.65 (IC 95\%: 0.54-0.74).

\section{UTILIDAD DE LOS ESTUDIOS DE IMAGEN EN EII}

EN LOS PACIENTES CON DIAGNÓSTICO ESTABLECIDO DE ENFERMEDAD INFLAMATORIA INTESTINAL Y DOLOR ABDOMINAL AGUDO CON SOSPECHA DE PERFORACIÓN SE DEBE REALIZAR UNA TC

GRADE 1 (recomendación fuerte, calidad de la evidencia alta).

\section{EN PACIENTES CON COLITIS ULCEROSA GRAVE SE RECOMIENDA REALIZAR PLACA SIMPLE DE ABDOMEN PARA DESCARTAR LA PRESENCIA DE MEGACOLON TÓXICO}

GRADE 1A (recomendación fuerte, calidad de la evidencia alta).

La perforación libre espontánea es un evento raro pero grave en la EC. Puede ser el resultado de una inflamación severa y es más común en la CU grave. Se estima que aproximadamente del 1 al $2 \%$ de los pacientes con EC presentarán una perforación libre inicialmente $o$ en algún momento durante el curso de la enfermedad. La tomografía computarizada (TC) es una herramienta importante para el diagnóstico de perforación en pacientes con dolor abdominal agudo y diagnóstico establecido de Ell. Un estudio observó que, entre 18 pacientes con megacolon tóxico, las TC revelaron complicaciones abdominales en cuatro pacientes, que habían sido omitidos tanto clínicamente como por hallazgos en placa simple de abdomen.

El diagnóstico de megacolon tóxico generalmente se realiza mediante signos clínicos de toxicidad sistémica respaldados por la confirmación por imagen. La detección de la dilatación del colon transverso $>5.5 \mathrm{~cm}$ por medio de una placa simple de abdomen sigue siendo la definición radiológica más reconocida y el método diagnóstico de megacolon tóxico más efectivo ${ }^{37}$.

\section{LA USG ABDOMINAL, LA TC Y LA RM SON ÚTILES PARA EVALUAR LA ACTIVIDAD Y COMPLICACIONES DE LA eNFERMEDAD DE CROHN}

GRADE 1A (recomendación fuerte, nivel de evidencia alta).

En el momento del diagnóstico, el $15.5 \%$ de los pacientes con EC tienen lesiones penetrantes (fístulas, flemones o abscesos). La ultrasonografía (USG), Ia TC y la resonancia magnética (RM) tienen una alta precisión para la evaluación de complicaciones penetrantes y para monitorear la progresión de la enfermedad ${ }^{38}$.

Panes, et al..$^{39}$, en una revisión sistemática, demostraron la utilidad de la USG para la evaluación de la EC, encontrando $S$ que van del 63 al 100\% y E del 77 al $100 \%$ para el diagnóstico de EC ileocolónica. En la misma revisión los autores observaron que la $S$ y la $E$ de la TC para el diagnóstico de fístulas, comparado con cirugía y endoscopia como estándar de referencia, fue del 70 y el $97 \%$ respectivamente.

La RM es muy precisa para la detección de abscesos, fístulas e inflamación transmural en la EC. Los 
resultados de cuatro estudios con un estándar de referencia adecuado (endoscopia y/o cirugía) mostraron que la RM tiene una $S$ para el diagnóstico de fístulas del $76 \%$ y una $\mathrm{E}$ del $96 \%$. La RM con enteroclisis parece ser la herramienta más eficaz para detectar fístulas intraabdominales ${ }^{40}$. La S y E de la RM para evaluar la actividad se investigaron en ocho estudios utilizando la colonoscopia como estándar de referencia. Se informó una alta correlación entre la actividad endoscópica y los hallazgos de la RM $(p=0.001)$. Para fístulas profundas, la RM con enteroclisis y la TC son preferibles a la USG. La USG y la TC están ampliamente disponibles y facilitan el drenaje temprano de abscesos ${ }^{41}$.

El score MaRIA (Magnetic Resonance Index of Activity) brinda una cuantificación de la actividad de la EC en los estudios de enterorresonancia. Los predictores que se asocian con actividad endoscópica son engrosamiento de la pared, realce del contraste, edema y úlceras. La puntuación oscila entre 0 y 12, por lo que se considera enfermedad en remisión con valores $\leq 2$, actividad leve para un índice de 3-6 y actividad moderada-grave si la suma $\geq 7^{42}$.

La elección del método de imagen dependerá de la disponibilidad, experiencia de la institución y la presentación clínica del paciente.

\section{Papel de la endoscopia en el diagnóstico enfermedad inflamatoria intestinal (indicaciones, contraindicaciones, biopsias, escalas de actividad endoscópica de la enfermedad, diferencias endoscópicas en colitis ulcerosa y enfermedad de Crohn)}

\section{Ante la sospecha de enfermedad inflamatoria intestinal, la IC con biopsias regionales es el estudio de elección para establecer el diagnóstico y extensión de la enfermedad}

GRADE 1A (recomendación fuerte, calidad de evidencia alta).

La ileocolonoscopia (IC) es un procedimiento seguro, con muy pocas contraindicaciones y debe realizarse en todos los pacientes con sospecha de Ell; se considera el estándar de oro para obtención de biopsias y estadificación de la enfermedad, seguimiento y descartar otros diagnósticos diferenciales. En pacientes en los que se sospecha Ell es importante realizar un examen meticuloso de la región perianal durante o previo
Tabla 4. Características endoscópicas de la enfermedad inflamatoria intestinal

\begin{tabular}{|l|l|}
\hline Colitis ulcerosa & Enfermedad de Crohn \\
\hline Eritema & Eritema \\
\hline Friabilidad & Friabilidad \\
\hline Pseudopólipos & Pseudopólipos (poco común) \\
\hline Afección rectal & Afección rectal (poco común) \\
\hline Daño continuo & Inflamación en parches \\
\hline Pérdida de la arquitectura & Imagen en empedrado \\
\hline Pérdida del patrón vascular & Pérdida de las vellosidades \\
\hline Erosiones & Estenosis \\
\hline Úlceras & Úlceras lineales y \\
serpentiginosas
\end{tabular}

Adaptada de Flynn, et al., $2019^{45}$ y Spiceland, et al., $2018^{46}$.

a la evaluación endoscópica, en búsqueda de fisuras, abscesos o fístulas ${ }^{43,44}$.

La canulación ileal con toma de biopsias es fundamental para diferenciar la EC de la CU (Tabla 4). Se debe considerar que puede existir ileítis por reflujo, la cual ocurre de un $17-25 \%$ de los pacientes con pancolitis en la CU. Se pueden identificar estenosis en hasta el $11 \%$ de los pacientes con EC en la evaluación endoscópica inicial, y pueden tener hasta un $24 \%$ de riesgo de malignidad en $\mathrm{CU}$ no tratada y un $6 \%$ en $\mathrm{EC}^{45,46}$.

\section{La esofagogastroduodenoscopia es útil en aquellos pacientes con sospecha de enfermedad inflamatoria intestinal $y$ síntomas gastrointestinales superiores}

GRADE 2B (recomendación débil, calidad de evidencia moderada).

En EC la afección proximal al ligamento de Treitz (esófago, estómago y duodeno) ocurre en aproximadamente un $16 \%$, pudiendo observar eritema, aftas, úlceras, estenosis y orificios fistulosos. Si bien existe una fuerte correlación entre daño del tracto gastrointestinal superior e ileítis terminal, colopatía y afección perianal, no se recomienda la realización sistemática de endoscopia superior en el adulto, sino únicamente en pacientes con síntomas digestivos superiores. 
Cuando se realiza, es indispensable la toma de biopsias para el diagnóstico histológico. Las alteraciones microscópicas más frecuentes incluyen: granulomas (40$68 \%$ ), inflamación crónica y alteración en las vellosidades intestinales. Se recomienda la obtención de al menos dos biopsias de esófago, estómago y duodeno $0^{43,44,46,47}$.

\section{La CE es un método útil en el abordaje diagnóstico de la enfermedad inflamatoria intestinal}

GRADE 2B (recomendación fuerte, calidad de evidencia moderada).

La afectación del intestino delgado (ID) ocurre en aproximadamente $80 \%$ de los pacientes con EC, y en aproximadamente un tercio de ellos la afectación será únicamente del ID ${ }^{48}$.

La CE tiene un alto rendimiento diagnóstico en pacientes con EC establecido o sospechoso, puede ser útil para identificar lesiones superficiales no detectadas por endoscopia y por radiología. Los hallazgos incluyen eritema, atrofia de las vellosidades intestinales, erosiones y estenosis. Un metaanálisis demostró que la CE tiene un rendimiento diagnóstico superior al observar involucro de intestino delgado en EC sospechosa y establecida en comparación con enterotomomografía (ETAC), enteroresonancia magnética (ERM) e IC. La ausencia de hallazgos de EC en CE está asociada con un valor predictivo negativo del 96 al 100\%. Se recomienda el uso de CE para evaluación adicional de síntomas gastrointestinales inexplicables, como en terapia con inmunosupresión o biológicos. En estudios retrospectivos y prospectivos más pequeños sugieren que, aunque puede ayudar a reclasificar una proporción de pacientes con Ell como EC, el manejo clínico no se ve afectado y la ausencia de hallazgos en CE no excluye el diagnóstico de EC. Hay evidencia limitada para indicar CE en la evaluación preoperatoria de pacientes con $\mathrm{CU} O$ antes de la anastomosis anal de bolsa ileal ${ }^{49}$. El desarrollo de una nueva CE (Crohn Capsule Given I) sugiere su eficacia para evaluar respuesta al tratamiento en pacientes con EC.

\section{La enteroscopia tiene un papel limitado en la evaluación inicial de pacientes con enfermedad inflamatoria intestinal conocida o sospechada}

GRADE 2B (recomendación fuerte, calidad de evidencia moderada).

La enteroscopia tiene un papel limitado en la evaluación inicial de pacientes con Ell conocida o sospechada debido a los altos rendimientos diagnósticos de modalidades menos invasivas como CE y estudios de imagen. En aquellos casos donde se reporten anormalidades en los estudios de imagen o CE, realizar la enteroscopia, permite la evaluación endoscópica e histológica, intervenciones terapéuticas como hemostasia, dilatación de estenosis o recuperación de cuerpo extraño $0^{49}$.

\section{Se recomienda el uso de polietilenglicol como preparación para la colonoscopia diagnóstica y de seguimiento en pacientes con enfermedad inflamatoria intestinal}

GRADE IA (recomendación fuerte, calidad de la evidencia alto).

Cuando se realiza colonoscopia para diagnóstico o seguimiento de la Ell, la preparación debe ser exhaustiva, efectiva, bien tolerada y segura. Hay que tener en cuenta que algunas preparaciones intestinales pueden inducir daño a la mucosa, pudiéndose atribuirse erróneamente a actividad de la enfermedad. De acuerdo con un estudio controlado, prospectivo, que compara tres preparaciones intestinales diferentes, el riesgo de producir lesiones similares a la Ell es diez veces más frecuente con el fosfato de sodio o soluciones a base de picosulfato que las de polietilenglicol (3.5 vs. $0.3 \%$; $p=0.03)^{50}$.

En casos de colitis fulminante, megacolon tóxico o colitis severa está contraindicada la colonoscopia por alto riesgo de perforación

GRADE $1 B$ (recomendación fuerte, nivel de evidencia moderado).

La IC es un procedimiento seguro, con baja tasa de complicaciones en pacientes con Ell. Sin embargo, se contraindica en pacientes con actividad severa. La perforación visceral espontánea, particularmente colónica, puede ocurrir en la Ell con actividad severa. La incidencia de perforación en una colonoscopia ordinaria es del 0.005 al $0.63 \%$. En algunos casos en los que está contraindicada la colonoscopia, la sigmoidoscopia flexible puede proveer una alternativa segura que permita la valoración distal del intestino y obtener biopsias. Adicionalmente la sigmoidoscopia flexible puede realizarse en pacientes con Ell establecida para determinar la actividad de la enfermedad, antes de iniciar terapias de rescate con inmunomodulador o biológico, en aquellos con 
enfermedad refractaria a tratamiento convencional y para descartar alguna infección concomitante (p. ej., CMV, Clostridioides o colitis isquémica $)^{46,51}$.

\section{Para el diagnóstico de enfermedad de Crohn y colitis ulcerosa se deben de tomar múltiples biopsias de los cinco segmentos del colon e íleon terminal}

GRADE $1 A$ (recomendación fuerte, nivel de evidencia alto).

El diagnóstico de Ell (CU y EC) debe establecerse mediante la combinación de la historia y evaluación clínica. El examen histológico de las biopsias intestinales sigue siendo un elemento clave en el estudio y diagnóstico de pacientes afectados, particularmente en la diferenciación de EC y CU.

Para realizar un diagnóstico confiable de Ell se requiere de tomar un mínimo de dos biopsias de cada uno de los cinco segmentos del colon (ascendente, transverso, descendente, sigmoides y recto) e íleon terminal independientemente de la apariencia macroscópica de la mucosa (normal o afectada). Las biopsias de cada segmento deben colocarse en recipientes con formol al 10\% separados y rotulados refiriendo el sitio de toma. Las muestras de biopsia deben ir acompañadas de información clínica que incluya hallazgos endoscópicos, así como la edad del paciente, la duración de la enfermedad y el tipo de tratamiento, las comorbilidades e historial de viajes ${ }^{52}$.

\section{Se recomienda la Escala de índice de severidad colonoscópica en colitis ulcerosa para valorar actividad en colitis ulcerosa y la extensión con la clasificación de Montreal}

GRADE 1A (recomendación fuerte, nivel de evidencia alto).

La escala de Mayo y la subescala endoscópica de Mayo que evalúan la actividad de la enfermedad tienen gran variabilidad interobservador, por lo que actualmente se recomienda utilizar el índice de severidad colonoscópica en CU (UCEIS, Ulcerative Colitis Endoscopic Index of Severity). EI UCEIS es el primer índice validado para la evaluación endoscópica global de actividad por medio de los hallazgos observados con endoscopios de alta resolución. Se describen los patrones vasculares, presencia de sangrado y ulceraciones o erosiones severas. Ha demostrado disminución de la variabilidad interobservador en la evaluación de la mucosa, pero no en la extensión (Tabla 5).
En la CU la extensión de la inflamación endoscópica puede ser clasificada con la clasificación de Montreal, como proctitis (E1), colitis izquierda (hasta la flexura esplénica) (E2) o colitis extensa (más allá de la flexura esplénica) (E3) ${ }^{4-46,53-55}$.

\section{Se recomienda la Escala endoscópica simple para enfermedad de Crohn en la evaluación endoscópica de la enfermedad de Crohn luminal}

GRADE $1 B$ (recomendación alta, nivel de evidencia moderado).

Los sistemas de puntuación endoscópica en la EC tienen como objetivo traducir la evaluación de la actividad de la enfermedad de la mucosa en un valor cuantificado. Este valor proporciona un registro claro y objetivo de la gravedad de la actividad endoscópica y permite normar la conducta terapéutica. El objetivo endoscópico final del tratamiento es la cicatrización de la mucosa ${ }^{56}$.

Se han desarrollado numerosos sistemas de clasificación y puntuación para estandarizar la evaluación endoscópica ${ }^{57,58}$.

La escala endoscópica simple para EC (SES-CD) se diseñó con el objetivo de poder servir tanto en la práctica clínica como en la investigación, y es una herramienta sencilla y cómoda. Este puntaje ha sido validado y ha demostrado una excelente correlación con el Crohn's Disease Endoscopic Index of Severity (CDEIS), con la actividad clínica y los biomarcadores ${ }^{59,60}$ (Tabla 6).

Sin embargo, al ser similar al CDEIS tiene las mismas limitaciones, ya que tiende a subestimar la gravedad de afectaciones más cortas, a la enfermedad aislada del íleon, y no existe un punto de corte que defina remisión o cicatrización de la mucosa, aunque comúnmente se acepta que un puntaje entre 0 y 2 puntos es indicativo de enfermedad endoscópicamente inactiva.

\section{El índice de Rutgeerts predice las posibilidades de recurrencia clínica en pacientes posquirúrgicos}

GRADE 1 A (recomendación alta, nivel de evidencia alto).

El índice de Rutgeerts clasifica a los pacientes intervenidos de resección ileocecal basándose en las lesiones endoscópicas que presentan seis meses posteriores a la cirugía y puede predecir las posibilidades de recurrencia clínica (Tabla 7). A pesar de que nunca ha llegado a ser validado, se acepta que aquellos pacientes que presentan unas lesiones más graves deben recibir tratamiento 
Tabla 5. Escala de índice de severidad colonoscópica en colitis ulcerosa ${ }^{44}$

\begin{tabular}{|l|l|l|}
\hline Lesiones & Puntuación & Definición \\
\hline Patrón vascular & $\begin{array}{l}\text { Normal (0) } \\
\text { Pérdida focal (1) } \\
\text { Ausencia completa (2) }\end{array}$ & $\begin{array}{l}\text { Patrón normal } \\
\text { Patrón vascular en parche } \\
\text { Ausencia de patrón vascular }\end{array}$ \\
\hline Hemorragia & $\begin{array}{l}\text { No (0) } \\
\text { Mucosa (1) } \\
\text { Luminal mínima (2) } \\
\text { Luminal moderada o severa (3) }\end{array}$ & $\begin{array}{l}\text { Sin sangre visible } \\
\text { Puntos o hilos de sangre coagulada superficial de fácil lavado } \\
\text { Algo de sangre líquida en la luz intestinal } \\
\text { Sangrado franco }\end{array}$ \\
\hline Erosiones y úlceras & $\begin{array}{l}\text { No (0) } \\
\text { Erosiones (1) } \\
\text { Úlceras superficiales (2) } \\
\text { Úlceras profundas (3) }\end{array}$ & $\begin{array}{l}\text { Sin lesiones } \\
<5 \text { mm blancos o amarillos }\end{array}$ \\
\hline $\begin{array}{l}\text { Úlceras }>5 \text { mm, con fibrina, superficiales } \\
\text { Úlceras profundas con bordes sobreelevados }\end{array}$
\end{tabular}

Adaptada de Klinger, et al., $2019^{44}$.

Tabla 6. Escala simple para la enfermedad de Crohn (SES-CD)*

\begin{tabular}{|c|c|c|c|c|}
\hline Variable & 0 & 1 & 2 & 3 \\
\hline Tamaño de la úlcera & No & Úlceras aftosas $(0.1-0.5 \mathrm{~cm})$ & $\begin{array}{l}\text { Úlceras grandes } \\
(0.5-2 \mathrm{~cm})\end{array}$ & $\begin{array}{l}\text { Úlceras muy grandes } \\
(>2 \mathrm{~cm})\end{array}$ \\
\hline Superficie de la úlcera & No & $<10 \%$ & $10-30 \%$ & $>30 \%$ \\
\hline Superficie afectada & Ninguna & $<50 \%$ & $50-75 \%$ & $>75 \%$ \\
\hline Estenosis & No & Única con paso & $\begin{array}{l}\text { Múltiples } \\
\text { Franqueable }\end{array}$ & Infranqueable \\
\hline
\end{tabular}

${ }^{*}$ SES-CD (rango 0-60) = suma de la puntuaciones en el íleon, colon derecho, transverso, izquierdo y recto para cada variable (rango 0-15 puntos en cada segmento). Adaptada de Schoepfer, et al., $2010^{59}$.

inmunomodulador, incluso si se encontraran asintomáticos, ya que las posibilidades de presentar recurrencia clínica y complicaciones a corto plazo son elevadas ${ }^{61,62}$.

\section{Seguimiento endoscópico de la enfermedad inflamatoria intestinal}

\section{La cicatrización de la mucosa debe determinarse endoscópicamente 3-6 meses después del inicio del tratamiento en pacientes con colitis ulcerosa y a los 6-9 meses en pacientes con enfermedad de Crohn}

GRADE $1 B$ (recomendación fuerte, calidad de evidencia moderada).

El concepto del «tratamiento por objetivos» (T2T, treat to target) fue desarrollado por el programa STRIDE (Selecting Therapeutic Targets in Inflammatory Bowel Disease), desarrollado por un grupo de expertos establecidos por la Organización Internacional para el
Estudio de las Enfermedades Inflamatorias Intestinales (IOIBD). EI T2T implica la identificación de un objetivo terapéutico predefinido, inicio y optimización del tratamiento, así como monitorización regular hasta que se logra el objetivo; todo de acuerdo con el paciente y en el contexto de sus necesidades individuales ${ }^{63}$.

Se ha demostrado que la cicatrización de la mucosa correlaciona con una respuesta clínica sostenida y disminución de los factores asociados a morbilidad, como el uso de corticosteroides, hospitalización, cirugía y CCR asociado a Ell ${ }^{63}$.

En un metaanálisis que incluyó 13 estudios con 2,073 pacientes con CU activa, los pacientes que lograron cicatrización de la mucosa en la primer evaluación endoscópica después de iniciar el tratamiento tuvieron una odds ratio (OR) de 4.5 (IC 95\%: 2.129.52) para lograr remisión clínica a largo plazo (después de por lo menos 52 semanas), OR de 4.15 (IC 95\%: 2.53-6.81) para evitar colectomía y OR de 8.4 (IC 95\%: 3.13-22.53) para lograr cicatrización de la 
Tabla 7. Índice de Rutgeerts

\begin{tabular}{|c|c|c|c|}
\hline Grado & Hallazgo endoscópico & Recurrencia & Endoscopia \\
\hline i0 & No lesiones & $<10 \%$ a los 10 años & \\
\hline i1 & $<5$ lesiones aftosas & $<10 \%$ a los 10 años & \\
\hline ¡2 & $\begin{array}{l}\text { ¡2a - lesiones confinadas a la anastomosis } \\
\text { ¡2b - } 5 \text { lesiones aftosas con mucosa normal entre lesiones: } \\
\text { áreas salteadas de lesiones más grandes }\end{array}$ & $40 \%$ a los 5 años & \\
\hline i3 & Ileítis aftosa difusa sobre mucosa inflamada & $70 \%$ al primer año & \\
\hline i4 & $\begin{array}{l}\text { Inflamación difusa con úlceras grandes nódulos y/o } \\
\text { estenosis }\end{array}$ & $70 \%$ al primer año & \\
\hline
\end{tabular}

Adaptada de Rutgeerts, et al., $1990^{61}$.

mucosa a largo plazo, así como una OR de 9.7 (IC 95\%: 0.94-99.67) para lograr una remisión clínica libre de esteroides, comparado con aquellos pacientes que no lograron cicatrización de la mucosa ${ }^{64}$. En pacientes con CU sintomáticos la evaluación endoscópica se recomienda a los tres a seis meses.

Otro metaanálisis mostró resultados similares en pacientes con EC, observando que aquellos pacientes que lograron cicatrización de la mucosa tuvieron un OR de 2.8 ( IC 95\%: 1.91-4.1) para lograr remisión clínica a largo plazo, una OR de 2.22 (IC 95\%: 0.86-5.69) para evitar una cirugía relacionada con la EC, y de 14.3 (IC 95\%: 5.57-36.74) para cicatrización de la mucosa a largo plazo ${ }^{65}$.

El programa STRIDE establece que, en la EC, el objetivo endoscópico más importante es lograr la ausencia de úlceras y que la evaluación endoscópica debe realizarse de seis a nueve meses después de iniciar el tratamiento66.

En la mayoría de los ensayos clínicos para evaluar las terapias biológicas se sugiere una evaluación de la mucosa al final de la fase de inducción, lo que se traduce en eficacia del medicamento. Bajo esta premisa, se sugiere que la evaluación endoscópica se realice de a los tres a seis meses del inicio del tratamiento.

\section{Se sugiere realizar reevaluación endoscópica con colonoscopia en casos de recaída severa, actividad persistente, síntomas nuevos o inexplicables y antes del cambio de terapia}

Grade 2c (recomendación débil, calidad de evidencia baja).

En casos de recaída, es necesario evaluar la severidad y extensión de la enfermedad, así como descartar factores desencadenantes (p. ej., infecciones $)^{40}$. Se puede realizar colonoscopia con toma de biopsias en búsqueda de infecciones oportunistas (CMV y Clostridioides entre otras $)^{52}$ En el caso particular de la infección por CMV, el uso de la histología/inmunohistoquímica para su detección es superior a la reacción en cadena de la polimerasa de CMV en sangre ${ }^{67}$.

La Ell es una enfermedad progresiva, por lo que en pacientes con enfermedad localizada inicialmente, con pérdida subsecuente de la respuesta al tratamiento, debe descartarse progreso en la extensión de la enfermedad 68 . En un estudio de seguimiento de 1,116 pacientes con CU se observó que más del $46 \%$ de los pacientes con proctitis y el $70 \%$ de los pacientes con colitis izquierda pueden desarrollar colitis extensa en el seguimiento ${ }^{69}$.

\section{La toma de biopsias en el seguimiento del enfermedad inflamatoria intestinal debe incluir 2 biopsias de cada uno de los 6 segmentos explorados durante la colonoscopia (5 segmentos colónicos e íleon terminal)}

GRADE $1 B$ (recomendación fuerte, calidad de evidencia moderada).

El tercer consenso Europeo del 2017 recomienda obtener al menos dos biopsias de cada segmento colónico (colon ascendente, colon transverso, colon descendente, sigmoides y recto) e íleon terminal, no solo de las áreas activas endoscópicamente, también de las áreas endoscópicamente normales, ya que el tratamiento puede inducir cambios histológicos ${ }^{52,70}$.

Un estudio prospectivo observacional de 179 pacientes con CU demostró que solo el $7 \%$ de aquellos pacientes con remisión clínica, endoscópica e histológica, tuvo 
recaída en un seguimiento de 12 meses; el riesgo relativo (RR) de recaída fue de 3.5 (IC 95\%: 1.9-6.4; $p=0.0001$ ) en pacientes con actividad histológica severa ${ }^{71}$.

\section{En pacientes con colitis ulcerosa con actividad limitada a recto ya conocida, el seguimiento de la actividad puede hacerse con RSC flexible}

GRADE 1B (recomendación fuerte, calidad de evidencia moderada).

La rectosigmoidoscopia (RSC) flexible es inadecuada para el seguimiento de pacientes con EC (no colónica), $\mathrm{CU}$ con colitis extensa o colitis izquierda. Su utilidad se limita a casos donde la colonoscopia se considera de alto riesgo (colitis fulminante) (en cuyo caso se puede realizar RSC flexible y diferir la colonoscopia completa para cuando el paciente se encuentre en mejores condiciones), evaluación de actividad en pacientes con CU y sospecha de infecciones sobreagregadas o diagnóstico diferencial (Clostridioides difficile, CMV o colitis isquémica) en pacientes con síntomas recurrentes 0 persistentes 0 en pacientes con actividad limitada a recto ${ }^{68}$.

Colombel, et al..$^{72}$ realizaron un estudio de concordancia, estudiando 229 vídeos de pacientes incluidos en un ensayo clínico; evaluaron la actividad endoscópica de 119 pacientes a la semanas 0,6 y 10 de tratamiento con etrolizumab. Cada vídeo se revisó dos veces y después de excluir videos incompletos o con registro de menos de dos segmentos, se realizaron 441 lecturas (por dos observadores expertos); en la evaluación inicial el $75 \%$ de los pacientes tenían actividad proximal al rectosigmoides. Hubo una fuerte correlación entre las dos técnicas (colonoscopia vs. RSC) para detectar cicatrización de la mucosa ( $\kappa: 0.84$, r: 0.85 ). Solo un vídeo mostró cicatrización endoscópica rectal con lesiones en colon proximal (9\%). En la evaluación endoscópica se confirmó mayor afectación en rectosigmoides comparada con colon proximal (UCEIS: $3.98 \pm 1.82$ vs. 2.38 \pm 1.98 ; $p \leq 0.0001$ ). Los autores observaron que, al encontrarse una actividad más severa en rectosigmoides, la RSC por sí sola sería efectiva para la toma de decisiones, las cuales se basan no solo en la extensión, sino en la actividad endoscópica.

En pacientes con CU se ha demostrado una adecuada correlación entre los hallazgos endoscópicos de colonoscopia comparada con RSC flexible; en estos casos, la RSC resultó eficaz, cómoda y más económi$\mathrm{ca}^{73}$. Aunque aún no hay evidencia suficiente para recomendarlo en todos los casos, se considera que, en pacientes con actividad inicial limitada a recto, con buena respuesta clínica y bioquímica, puede ser una adecuada opción de seguimiento.

\section{Escrutinio de cáncer en enfermedad inflamatoria intestinal}

\section{Iniciar el escrutinio de CCR con} colonoscopia a partir de los 8 años de la aparición de los síntomas en todos los pacientes con colitis ulcerosa y enfermedad de Crohn con actividad colónica para evaluar la extensión de la enfermedad y excluir displasia

GRADE $1 A$ (recomendación fuerte, calidad de evidencia alta).

La presencia de Ell con inflamación colónica incrementa el riesgo de padecer CCR. Las estrategias de vigilancia han demostrado disminuir la incidencia de CCR a lo largo del tiempo. El riesgo de CCR en Ell incrementa con la duración y la extensión de la enfermedad, a una tasa de aproximadamente $0.5-1 \%$ por año después de 8-10 años del inicio de los síntomas.

Un estudio reportó una incidencia acumulada de CCR del $1 \%$ a los 10 años, el $3 \%$ a los 20 años y el $7 \%$ a los 30 años en pacientes con Ell ${ }^{74}$.

La vigilancia con programas de colonoscopia de escrutinio ha demostrado reducir la morbimortalidad asociada a CCR en este grupo de pacientes ${ }^{70}$.

Cuando la actividad se limita al recto, sin evidencia previa o actual de extensión proximal, la vigilancia endoscópica periódica no es necesaria. Una cohorte de 3,117 pacientes con CU evaluó el riesgo de CCR y observó una relación entre la extensión de la enfermedad y la tasa de incidencia, siendo 1.7 (IC 95\%: 0.83.2) para proctitis, 2.8 (IC 95\%: 1.6- 4.4) para colitis izquierda y 14.8 (IC 95\%: 11.4-18.9) para pancolitis. La edad y la extensión al momento del diagnóstico fueron factores de riesgo alto e independientes para $\mathrm{CCR}^{75}$.

La atipia reactiva (falso positivo para displasia) puede observarse en biopsias tomadas en pacientes con Ell activa, es por ello que la vigilancia colonoscópica debe realizarse en pacientes con $\mathrm{CU}$ en remisión ${ }^{52}$.

\section{En pacientes con colangitis esclerosante primaria se debe realizar colonoscopia anual independientemente de la actividad, extensión y duración de la enfermedad inflamatoria intestinal}

GRADE 1B (recomendación fuerte, calidad de evidencia moderada). 
Tabla 8. Recomendaciones de vigilancia con colonoscopia de acuerdo con el riesgo de cáncer colorrectal (CCR) en enfermedad inflamatoria intestinal

\begin{tabular}{|l|l|l|}
\hline Riesgo bajo & Riesgo moderado & Riesgo alto \\
\hline $\begin{array}{l}\text { Colitis extensa con inflamación } \\
\text { histológica o endoscópica leve } \\
\text { Colitis en } \leq 50 \% \text { del colon }\end{array}$ & $\begin{array}{l}\text { Colitis extensa con inflamación } \\
\text { histológica o endoscópica I } \\
\text { eve/moderada (o ambas) } \\
\text { CCR en un familiar de primer } \\
\text { grado mayor a } 50 \text { años }\end{array}$ & $\begin{array}{l}\text { Colitis extensa con inflamación histológica o endoscópica } \\
\text { severa (o ambas) }\end{array}$ \\
$\begin{array}{ll}\text { CCR en un familiar de primer grado menor de } 50 \text { años } \\
\text { Pistoria de CEP (incluidos los pacientes postrasplantados) }\end{array}$ \\
\hline Cada 5 años & Cada 2-3 años & Displasia diagnosticada en los últimos 5 años \\
\hline
\end{tabular}

CEP: colangitis esclerosante primaria.

Adaptado de Maaser, et al., $2019^{40}$.

La presencia de CEP es hasta el momento el factor de riesgo más importante para CCR en Ell ${ }^{76}$. En una cohorte de 58 pacientes con CEP y Ell (40 CU con pancolitis, 9 EC colónica y 9 Ell no clasificada) en seguimiento se observó un riesgo acumulado de desarrollar displasia/CCR del $9 \%$ a los 10 años, el 31\% a los 20 años y el $50 \%$ después de los 25 años vs. 2, 5 y $10 \%$ respectivamente en el grupo control (pacientes con Ell sin CEP) ${ }^{77}$.

Los intervalos de vigilancia deben basarse en los factores de riesgo de cada paciente ${ }^{52,40,68,70}$ (Tabla 8).

\section{Los pacientes con enfermedad inflamatoria intestinal y estenosis diagnosticada en los primeros 5 años de la enfermedad deben someterse a vigilancia endoscópica anual}

GRADE 2B (recomendación débil, calidad de evidencia moderada).

Se ha observado que la presencia de estenosis incrementa el riesgo de CCR tanto en CU como en EC ${ }^{40}$. Se debe obtener biopsia de cualquier estenosis para descartar malignidad. La presencia de estenosis colónica se asocia al 3.6-4.9\% de probabilidad de CCR en 5-10 años de seguimiento ${ }^{78}$.

Los pacientes con estenosis diagnosticadas en los últimos cinco años deben someterse a vigilancia anual con toma de biopsias, idealmente, antes de realizar la dilatación con balón ${ }^{40}$. El estudio GETAID (Groupe d'Étude Thérapeutique des Affections Inflammatoires du Tube Digestif) reportó displasia o cáncer en el 3.5\% de los pacientes con Ell que tenían el antecedente de cirugía por estenosis colónica ${ }^{79}$.

En casos de estenosis en íleon y/o intestino delgado, que son los sitios más frecuentemente afectados por la EC, la conducta de vigilancia que seguir no es clara, por lo que cada caso deberá individualizarse.

\section{Las biopsias dirigidas por cromoendoscopia incrementan la tasa de detección de displasia}

GRADE 1A (recomendación fuerte, calidad de evidencia alta).

Un ensayo clínico demostró que la detección de CCR con biopsias dirigidas no es inferior que cuando se toman aleatoriamente ${ }^{80}$.

En la actualidad se considera que en la mayor parte de los casos de displasia detectada durante la endoscopia convencional con luz blanca y biopsias al azar, la imagen mejorada facilita la detección de displasia ${ }^{81}$. El método preferido actual de vigilancia es la cromoendoscopia de superficie con colorantes, acompañado de biopsias dirigidas y/o resección de lesiones visibles $^{40,52,70}$. Los agentes de contraste que se utilizan con mayor frecuencia incluyen azul de metileno al $0.1 \%$ o índigo carmín con diluciones entre 0.03 y el $0.5 \%$. La preparación del colon debe ser excelente para permitir la evaluación detallada de la mucosa. El colonoscopio debe insertarse hasta el ciego con luz blanca y durante el retiro el colorante debe rociarse por segmentos, aspirando el exceso de líquido. La cromoendoscopia resalta los detalles de la mucosa como la nodularidad y las anormalidades topográficas, así como las elevaciones y las depresiones. Cuando se identifica una lesión, la cromoendoscopia ayuda a definir la morfología de la lesión, su tamaño, bordes y datos sugestivos de invasión submucosa ${ }^{82,83}$. Las lesiones que se considere que pueden resecarse se deberán resecar, o deben tatuarse y referirse a un centro con experiencia para realizar resección 
endoscópica de la mucosa (REM) o disección ezca de la submucosa (DES). Debe tomarse biopsias dirigidas de las lesiones que se crea que no pueden resecarse o de significado incierto. Incluso si no se identifican lesiones, deben tomarse dos biopsias de cada segmento para determinar la extensión del daño histológico y la severidad de la enfermedad ${ }^{40,81}$.

La cromoendoscopia de todo el colon se ha comparado prospectivamente frente a la endoscopia de alta definición con resultados del $21.3 \%$ de la cromoendoscopia vs. el $9.3 \%$ de la endoscopia de alta resolución, con una ventaja del $120 \%$ a favor de la cromoendoscopia $(p=0.004)^{84}$. Las biopsias al azar solo valoran menos del $1 \%$ de la mucosa total ${ }^{82}$. Un metaanálisis de estudios prospectivos comparando la cromoendoscopia y la endoscopia convencional con luz blanca mostró mejoría en un 7\% (IC 95\%: 3.3-10.3\%) en la detección de displasia o cáncer para la cromoendoscopia ${ }^{85}$.

En contraste con la cromoendoscopia de superficie, la imagen de banda estrecha y las tecnologías de cromoendoscopia digital que usan filtros para aumentar el contraste de la mucosa y la vasculatura no han demostrado incremento en la detección de neoplasia.

La presencia de displasia (de alto o bajo grado) debe ser confirmada por un segundo patólogo experto en patología gastrointestinal ${ }^{40}$, ya que existe un significativo grado de variabilidad en la interpretación del grado de displasia incluso entre patólogos expertos en aparato digestivo ${ }^{86}$.

Un paciente con displasia de bajo grado confirmada, detectada en mucosa sin lesiones endoscópicas visibles, deberá tener un control de colonoscopia con cromoendoscopia y biopsias aleatorias a los tres meses ${ }^{40}$.

\section{Manejo endoscópico de las complicaciones de la enfermedad inflamatoria intestinal y situaciones especiales}

\section{La enfermedad inflamatoria intestinal activa o en tratamiento con esteroides sistémicos incrementa el riesgo de desarrollar complicaciones}

GRADE $1 C$ (recomendación fuerte, calidad de evidencia baja).

El papel de la endoscopia en las Ell ha cobrado gran relevancia en los últimos años en el tratamiento de las complicaciones. Las aplicaciones principales de la endoscopia terapéutica en pacientes con Ell incluyen la dilatación con balón de estenosis benignas asociadas y la REM de lesiones displásicas polipoides o no polipoides ${ }^{87}$.

La perforación visceral puede ocurrir de forma espontánea por la severidad de la enfermedad o como complicación en el momento de la endoscopia diagnóstica o terapéutica. La incidencia de perforaciones en la colonoscopia ordinaria sin Ell va del 0.005 al $0.2 \%{ }^{51,88}$. Sin embargo, la literatura acerca de la prevalencia y factores de riesgo en población con Ell es limitada.

Un estudio poblacional basado en datos de Medicare demostró una tasa de perforación del $0.06 \%$ en pacientes con Ell ${ }^{89}$. El grupo de Gastroenterología y Endoscopia de la Cleveland Clinic determinó en un estudio retrospectivo que la prevalencia de perforación en pacientes con Ell era del $1 \%$ comparado con el $0.6 \%$ en el grupo control. En este estudio se reportaron la edad mayor a 65 años, el sexo femenino y la realización de dilatación endoscópica como factores de riesgo independientes para la perforación en el grupo de Ell, asimismo se demostró que la severidad de la enfermedad y el uso concurrente de corticosteroides se asociaban con un riesgo incrementado de perforación ${ }^{90}$.

Por otro lado, no se ha observado un incremento en las tasas de perforación en endoscopia diagnóstica o terapéutica en Ell, en pacientes con terapia combinada con inmunomoduladores ${ }^{91}$.

Recomendamos que la endoscopia terapéutica en los pacientes con Ell se realice en centros de alto volumen por endoscopistas con experiencia en el manejo de las complicaciones de estos pacientes, así como establecer un equipo multidisciplinario para potenciales complicaciones previo a realizar terapia endoscópica.

\section{El manejo endoscópico de la displasia en enfermedad inflamatoria intestinal debe ser realizado por un experto en técnicas de resección avanzada}

GRADE 1A (recomendación fuerte, calidad de evidencia alta).

Los avances en las herramientas de detección permiten que más áreas de displasia en pacientes con Ell sean visibles e identificables al momento del estudio (la displasia invisible representa un 10\% de los casos), permitiendo la valoración e identificación de aquellas que pueden ser manejadas endoscópicamente ${ }^{92}$.

La displasia visible (el $89 \%$ son elevadas) se clasifica endoscópicamente en resecable y no resecable ${ }^{93}$. El término resecable aplica a aquella lesión cuyos márgenes son identificables, con posibilidad a la 
inspección visual que sugiera la resección completa, que posteriormente pueda ser confirmada por histología y el tejido adyacente a la resección se encuentre libre de displasia. En todo momento se debe tratar de realizar REM en bloque y tomar biopsias del tejido adyacente (cuatro cuadrantes) para asegurar que los márgenes están libres de displasia, sobre todo si hay datos de invasión submucosa ${ }^{94}$.

Aunque se pueden lograr tasas de resección completa cuando se realiza REM en fragmentos, hasta en un 10 a un $25 \%$ de los casos puede haber recurrencia local a largo plazo. Cabe destacar que las lesiones París 0-lla o 0-lla+ls tienen bajo riesgo de invasión, aquellas $0-\|l a+c, 0-\| c$ o $0-I s+c$ tienen mayor riesgo de invasión submucosa ${ }^{95}$.

El manejo de la displasia de alto grado localizada puede ser REM o DES. Ambos han demostrado tasas de curación sin recurrencia de displasia a dos años de seguimiento. La REM está indicada en lesiones con displasia polipoide y no polipoide con márgenes claros $\mathrm{y}<20 \mathrm{~mm}$ (25 mm en colon izquierdo, principalmente recto). La DES está indicada en lesiones con displasia no polipoide, cuando con REM no es posible una resección en bloque, cuando hay sospecha de fibrosis y en lesiones con tamaño $>20 \mathrm{~mm}$. En un metaanálisis y revisión sistemática se demostró una incidencia anual del $0.5 \%$ de CCR en pacientes con Ell a quienes se les realizó resección de pólipos (0-Ip y 0 -Is), sin mayores eventos adversos ${ }^{96}$.

Suzuki, et al. ${ }^{97}$ reportaron disección en más del $94 \%$ de 32 pacientes con displasia no polipoide en $\mathrm{CU}$ con una media de tamaño de $33 \mathrm{~mm}$ (rango: $12-73 \mathrm{~mm}$ ) y con tasas de resección en bloque y R0 del 97 y $79 \%$ respectivamente. Yang, et al. ${ }^{98}$ reportaron tasas de resección R0 y en bloque en un 80 y 93.3\% respectivamente en 15 pacientes con lesiones de $23 \mathrm{~mm}$ (12-48 mm). La tasa de resección para DES en displasia asociada a CU se encuentra entre el 70 y el $80 \%{ }^{99}$. Las tasas de éxito y complicaciones en el abordaje endoscópico para displasia asociada a CU pueden ser comparables al manejo para CCR esporádico.

Son indicaciones para descartar el manejo endoscópico: presentar factores de riesgo para CCR, distribución multifocal de displasia de alto grado no visible y apariencia endoscópica de displasia visible no resecable (márgenes mal definidos, invasión submucosa, elevación asimétrica a la inyección, ulceraciones, o depresión larga y cambios neoplásicos planos con pérdida de arquitectura glandular adyacente a la lesión).

\section{Para el manejo de estenosis por enfermedad de Crohn se debe realizar su clasificación adecuada y, de acuerdo con esta, los métodos ideales para su manejo son la dilatación con balón y cortes radiales. La colocación de prótesis se reserva para casos específicos}

GRADE 1B (recomendación fuerte, calidad de evidencia moderada).

En pacientes con EC, el desarrollo de complicaciones es una condición que incrementa a lo largo del tiempo, si bien al diagnóstico el fenotipo inflamatorio es el más frecuente, hasta un $11 \%$ pueden iniciar con estenosis y a lo largo del tiempo se espera que estas ocurran en cerca de un $50 \%$, siendo su etiología multifactorial ${ }^{100}$.

Actualmente la endoscopia y la cirugía son las dos modalidades terapéuticas para las estenosis, sin embargo, las características inherentes al procedimiento quirúrgico, así como las complicaciones postoperatorias y recurrencia de la enfermedad han hecho que la endoscopia se posicione como una opción válida de tratamiento, considerada un puente entre el tratamiento médico y el quirúrgico ${ }^{101,102}$.

En la evaluación de este grupo de pacientes es importante contar con un estudio complementario de imagen (RM, TC, USG), no solo por su S y E cercanas al $90 \%$ para diagnosticar fístulas, sino también porque ayuda a establecer las características de las estenosis (fuente, localización, número, grado, tipo, longitud y condiciones asociadas como fístula y/o absceso), lo cual es necesario previo al procedimiento endoscópico ${ }^{103-105}$.

Lo anterior es importante, ya que las características de la estenosis determinan los resultados terapéuticos. Un estudio reportó que la longitud de la estenosis fue el único factor predictor de eficacia de la dilatación con balón a largo plazo, observándose que con cada $\mathrm{cm}$ de incremento en la longitud de la estenosis se asoció a cirugía temprana en un $8 \%$, por lo que los autores recomendaron un diámetro máximo de $5 \mathrm{~cm}$ para poder maximizar un periodo libre de cirugía ${ }^{106}$. En el intestino delgado, la evidencia actual sugiere que el límite de longitud de las estenosis que garantiza un mayor periodo libre de cirugía posterior a la dilatación con balón es de $\leq 2 \mathrm{~cm}^{107}$. De acuerdo con la localización, se ha visto que las estenosis de duodeno, a diferencia de las de yeyuno/íleon y colon, presentan un riesgo hasta cinco veces mayor de cirugía (RR: 4.7, $p=0.038$ y RR: $5.6, p=0.03$, respectivamente). 
En relación con el número de estenosis, en un estudio retrospectivo de pacientes con estenosis múltiples se encontró que la presencia de $>3$ estenosis se asoció con un mayor riego de cirugía, a diferencia de los pacientes con 2-3 estenosis (RM: 14.1, IC 95\%: 1.6-120.3 vs. RM: 1.5, IC 95\%: 0.8-2.8, respectivamente) ${ }^{108}$.

En cuanto a la efectividad de la dilatación con balón, existen diferentes estudios con resultados diversos, reportándose éxito técnico del $71-100 \%$ y efectividad a largo plazo del $50-100 \%$, mientras que la frecuencia con que se evita la intervención quirúrgica va del 28 al $52 \%$; lo anterior es debido a la falta de estandarización de la técnica, duración del seguimiento e inclusión a población heterogénea ${ }^{109}$.

Shivashankar, et al. ${ }^{110}$ reportaron la experiencia en 273 pacientes con EC y estenosis en quienes se realizaron un total de 601 procedimientos, de los cuales más del $90 \%$ fueron exitosos y en un $2.1 \%$ se observaron complicaciones, siendo la perforación la principal. Además observó que al menos una tercera parte de los pacientes requirieron reintervención endoscópica posterior en una mediana de seguimiento de 2.6 años (intervalo mínimo-máximo: 1.8-2.9 años), y la probabilidad acumulada de reintervención se incrementó con el paso del tiempo (33.6\% a 1 año, IC 95\%: 25.9$38.7 \%$; $53.9 \%$ a 3 años, IC 95\%: $45.9-61.2 \%$; y $60.2 \%$ a 5 años, IC 95\%: 51.4-67.5\%).

En un metaanálisis de 24 estudios (1,163 pacientes) se reportó un éxito a corto plazo (éxito técnico) cercano al $90 \%$, a largo plazo, con una mediana de seguimiento de 15 a 70 meses, se observó que la dilatación con balón en una sola sesión fue efectiva en el $44 \%$ de los pacientes, mientras que el resto necesitó dos o más sesiones. La frecuencia de cirugía posterior a dilatación fue menor en las estenosis asociadas a anastomosis que en las estenosis primarias (18 vs. $29 \%$ ), sin embargo, la asociación no fue significativa. En relación con la longitud de las estenosis, aquellas $\leq 4 \mathrm{~cm}$ se asociaron con un menor riesgo de intervención quirúrgica [RR: 0.48; IC 95\%: 0.26-0.90); $p=0.02]^{112}$.

En otro metaanálisis de 25 estudios (1,089 pacientes y 2,664 dilataciones) se reportó de forma global un éxito técnico del 90.6\% (IC 95\%: 87.8-92.8\%), éxito clínico del 70.2\% (IC 95\%: 60-70.8\%), complicaciones en el 6.4\% (IC 95\%: 5-8.2\%) y perforación en 3\% (IC 95\%: 2.2-4\%). En el análisis de subgrupo no hubo diferencias en los desenlaces entre las características de las estenosis (primarias y secundarias), así como en la actividad de la enfermedad ${ }^{112}$.

La dilatación gradual con balón se recomienda en diámetros de $18-20 \mathrm{~mm}$, incluso si se necesitan múltiples sesiones con la finalidad de evitar complicaciones. Lo anterior debido a los datos reportados en un estudio en el que se encontró que el calibre al cual se realizaba la dilatación no se asoció con incremento en la eficacia clínica, necesidad de reintervención endoscópica o cirugía.

Debido a que una proporción considerable de pacientes con estenosis requieren reintervención endoscópica o cirugía (> 30\%), se han desarrollado nuevos procedimientos endoscópicos como la electroincisión, la cual puede ser mediante técnica de estricturotomía o estricturoplastia; y la colocación de endoprótesis, en casos de estenosis refractarias.

En un estudio de 85 pacientes con múltiples estenosis (tratamiento de 127 estenosis), la electroincisión tuvo un éxito técnico del $100 \%$, requiriendo tratamiento quirúrgico el $15.3 \%$ de los pacientes seguidos durante un periodo de 0.9 años (rango intercuartílico [RIC]: 0.31.8) y con una mediana de dos procedimientos (RIC: 1-3), los efectos adversos se presentaron en el $3.7 \%$ de los $\operatorname{casos}^{113}$. El mismo grupo de investigadores reportó los resultados comparativos entre electroincisión y dilatación con balón en una cohorte retrospectiva (185 pacientes) de pacientes con EC y estenosis de la anastomosis (secundarias), encontrando éxito técnico en el $100 \%$ en los casos de electroincisión y el $89.5 \%$ en el grupo de dilatación con balón, así como necesidad de cirugía en el 9.5 y el $33.5 \%$ ( $p=0.03)$, respectivamente; sin embargo, el periodo de seguimiento entre ambos grupos fue diferente. En relación con los efectos adversos, el grupo de electroincisión presentó más hemorragia posprocedimiento y el de dilatación con balón, más perforación ${ }^{114}$.

Actualmente la evidencia es limitada en relación con el empleo de prótesis metálicas autoexpandibles o biodegradables, siendo principalmente la experiencia reportada en series de casos, en pacientes con estenosis refractarias y largas, sin existir hasta el momento un consenso en su empleo, tipo de prótesis y duración.

\section{El ultrasonido endoscópico es actualmente una herramienta accesible para evaluar la enfermedad perianal y sus complicaciones en enfermedad de Crohn}

GRADE 2B (recomendación débil, calidad de evidencia moderada).

El involucro anorrectal puede ocurrir hasta en un tercio de los pacientes con EC. Este va a ocasionar lesiones perianales que pueden ir de fisuras simples a 
abscesos y fístulas complejas que causan discapacidad y alteración en la calidad de vida de los pacientes ${ }^{115}$. La Asociación Americana de Gastroenterología (AGA) clasifica las fístulas como simples o complejas de acuerdo con su origen. La importancia de la clasificación de las fístulas radica en que tiene un valor pronóstico, ya que aquellos pacientes con fístulas simples pueden tener tratamientos menos agresivos y mejores desenlaces que los pacientes con fístulas complejas ${ }^{116}$.

El ultrasonido endoscópico transperineal (USTP) y la RM son las mejores modalidades para el diagnóstico y la evaluación de las fístulas complejas en la EC. Sin embargo, la RM es un método costoso, que puede ser poco accesible, y en niños con EC puede tener ciertas limitaciones ${ }^{117}$.

En el USTP las fístulas se observan como estructuras hipoecogénicas, sin embargo, el trayecto puede observarse hiperecogénico debido a aire en el interior. En centros de alto volumen se realiza aplicación de peróxido de hidrógeno dentro de la fístula para formación de burbujas que permiten mejor detección sonográfica ${ }^{51}$.

En un metaanálisis se evaluó el rendimiento diagnóstico del USTP. Se observó que la S para detectar fístulas perianales es del 98\% (IC 95\%: 96-100\%) y el valor predictivo positivo (VPP) del 95\% (IC 95\%: 90-98\%). La detección de aperturas internas tuvo una $\mathrm{S}$ del 91\% (IC 95\%: 84-97\%) con un VPP del 87\% (IC 95\%: 76-95\%). Por otro lado, el UTP tiene una S del 86\% (IC 95\%: 67$99 \%$ ) y un VPP del $90 \%$ (IC 95\%: 76-99\%) en la detección de abscesos perianales ${ }^{119,120}$.

En diversos estudios prospectivos con el objetivo de comparar la RM con el USTP en población adulta y pediátrica se ha demostrado que el USTP tiene precisión diagnóstica semejante a la RM para detectar abscesos pequeños y superficiales, así como para clasificar la enfermedad perianal, con una concordancia con la clasificación de la AGA del $86 \%$ ( $\mathrm{k}=0.83)$.

\section{Colangiografía}

\section{LA CRM ES EL MÉTOdO DE PREFERENCIA PARA EL DIAGNÓSTICO DE COLANGITIS ESCLEROSANTE PRIMARIA}

LA CRM ES EL MÉTODO DE PREFERENCIA PARA EL DIAGNÓSTICO DE COLANGITIS ESCLEROSANTE PRIMARIA (CEP). LA CPRE TIENE UN PAPEL RELEVANTE EN EL MANEJO Y DIAGNÓSTICO DE LAS ESTENOSIS INDETERMINADAS Y EL COLANGIOCARCINOMA. GRADE 1B (Recomendación fuerte, calidad de evidencia moderada).
En la actualidad se prefiere la colangiografía por resonancia magnética (CRM) para el diagnóstico de la CEP al ser un método no invasivo, con menos complicaciones y más barato ${ }^{121,122}$. La colangiopancreatografía retrógrada endoscópica (CPRE) se asocia con múltiples complicaciones infecciosas, como colecistitis, colangitis e infecciones asociadas al duodenoscopio, y las asociadas al procedimiento, como hemorragia, pancreatitis y perforación ${ }^{123}$. En un estudio de casos y controles se comparó de manera retrospectiva la precisión diagnóstica y el acuerdo interobservador de la CRM frente a la CPRE en pacientes con CEP, encontrando $S$ y $E$ similares por arriba del $81 \%$ entre los dos métodos diagnósticos sin diferencias significativas $(p=0.12)$ con buen acuerdo interobservador $(k$ para CRM de 0.83; para CPRE de 0.73$)^{124}$. El metaanálisis más grande con el que se cuenta en la actualidad en relación con la utilidad diagnóstica de la CRM incluyó seis estudios prospectivos controlados, reportando $S$ y E del 86 y el $94 \%$ respectivamente para el diagnóstico de CEP. Sin embargo, la CRM es menos sensible comparada con la CPRE en detectar cambios más tempranos asociados a la CEP y es menos específica en pacientes con cirrosis ${ }^{125}$.

La CEP al ser una enfermedad inflamatoria crónica y estenosante de la vía biliar se asocia a estenosis dominantes en un $36-50 \%$ y desarrollo de colangiocarcinoma (CCA) en un $20 \%{ }^{126}$. La definición de una estenosis dominante se refiere a una estenosis diagnosticada por CPRE con un diámetro menor a $1.5 \mathrm{~mm}$ en el conducto biliar común y menor de $1 \mathrm{~mm}$ en conductos hepáticos proximales a $2 \mathrm{~cm}$ de la confluencia del conducto hepático común que causa obstrucción biliar clínica y bioquímicamente significativa ${ }^{127,128}$. Ya que la prevalencia en los pacientes con CEP de estas dos entidades es alta y de gran importancia, al detectar una estenosis dominante se requiere un abordaje diagnóstico y terapéutico más amplio. La utilidad de la CPRE en estos escenarios se determinó en un estudio prospectivo de una cohorte de 171 pacientes con CEP y estenosis dominante con seguimiento a 20 años que demostró la supervivencia libre de trasplante del $81 \%$ a 5 años y del $52 \%$ a 10 años posterior a la primera intervención endoscópica, con una prevalencia del $6 \%$ de $\mathrm{CCA}^{129}$. Debido al limitado rendimiento diagnóstico $(40 \%)$ del cepillado biliar para citología y la relevancia del significado de las lesiones, en la actualidad ha tomado importancia la evaluación colangioscópica directa por operador único (CDOU) de dichas lesiones con opción a toma de biopsia. En un estudio observacional de una cohorte de 105 pacientes con estenosis 
indeterminadas, la precisión diagnóstica global de la colangioscopia directa sin toma de biopsia fue del $89.5 \%$ frente a un $83.2 \%$ para la toma de biopsia guiada por colangioscopia directa, siendo la experiencia del operador (menos de $25 \mathrm{CDOU}$ ) y los niveles de hiperbilirrubinemia, los que impactan negativamente la precisión diagnóstica de la $\mathrm{CDOU}^{130}$. En la actualidad, el mejor rendimiento diagnóstico para la evaluación de estas lesiones es la CDOU con visualización directa y toma de biopsia guiada. Para el tratamiento endoscópico por CPRE de estas lesiones se han sugerido diversos abordajes terapéuticos en casos de ictericia y colangitis como la dilatación con balón con o sin la colocación de prótesis plásticas por periodos cortos (10 días), con diversos resultados. En un ensayo clínico multicéntrico de pacientes con CEP y una estenosis dominante (65 pacientes), el uso de prótesis plásticas por periodos cortos no fue superior a la dilatación con balón y la primera se asoció de manera significativa con mayores tasas de complicaciones tales como pancreatitis y colangitis (45\% para el grupo de prótesis plásticas) vs. la dilatación con balón $(7 \%)^{131}$.

\section{Se recomienda EGD como parte de la evaluación inicial de niños con sospecha de enfermedad inflamatoria intestinal, independientemente de la presencia de síntomas gastrointestinales superiores}

GRADE $1 C$ (recomendación fuerte, calidad de evidencia baja).

El abordaje diagnóstico propuesto en los Criterios de Porto incluye el realizar una endoscopia gastroduodenal (EGD) como parte de la evaluación inicial; se recomienda sin importar la presencia de síntomas gastrointestinales, ya que se pueden identificar lesiones ulcerosas y en el estudio histopatológico encontrar principalmente granulomas de células gigantes o úlceras aftosas, lo que puede confirmar el diagnóstico de EC en un 11 a $29 \%$ de los casos, en los que, de no haberse realizado este estudio, se habría omitido. Además, se ha encontrado gastritis focal en EC e inflamación del tracto gastrointestinal superior en un $75 \%$ de los pacientes pediátricos con $\mathrm{CU}^{132}$.

Los criterios de Porto fueron revisados por la Sociedad Europea de Gastroenterología, Hepatología y Nutrición Pediátrica en 2014, continuando con la recomendación de realizar EGD a todos los pacientes con sospecha de Ell, independientemente de su sintomatología. Y agregando la recomendación de tomar múltiples biopsias (al menos dos por segmento visualizado), independientemente de las características macroscópicas ${ }^{133}$. En los resultados del registro EUROKIDS de cinco años, se identificó que en 1,811 pacientes con Ell y EGD inicial, el $35 \%$ de estos con EC presentó alteraciones macroscópicas, siendo específicas en un $24 \%$. Anormalidades histológicas establecieron el diagnóstico de EC en el $4.5 \%$ y además en el $3 \%$ se logró documentar un granuloma aislado ${ }^{134}$. Lemberg, et al..$^{135}$ evidenciaron en una serie retrospectiva de 76 pacientes pediátricos con diagnóstico final de Ell que la EGD confirmó el diagnóstico en el 14.6\% de los pacientes con histología colónica normal, tanto por los hallazgos macroscópicos como histológicos. Granulomas aislados para establecer el diagnóstico de EC se han reportado en distintas series, en entre un 2 y un $21 \%{ }^{136}$.

La utilidad de la EGD se ha documentado en la revisión cegada de biopsias iniciales de pacientes pediátricos con Ell, donde se encontró que un $11 \%$ de los niños con EC fueron diagnosticados por inflamación granulomatosa en el tracto gastrointestinal superior, otros hallazgos como gastritis localizada y criptitis focal duodenal se encontraron significativamente presentes en EC y al compararse con sujetos con CU y otros diagnósticos se estableció una S y VPP del 99 y el $93 \%$ respectivamente ${ }^{137}$.

Son pocos los estudios controlados y cegados realizados en relación con la EGD inicial en pacientes con Ell. Tobin, et al. ${ }^{138}$ realizaron un estudio transversal comparativo cegado de biopsias de tubo digestivo alto, en los que se enrolaron 28 casos con EC, 14 con CU y 22 controles con enfermedad por reflujo gastroesofágico, encontrando gastritis en el $92 \%$ de los pacientes con EC vs. $50 \%$ en controles ( $p<0.001$ ) y granulomas en un $40 \%(p<0.01)$, otros hallazgos encontrados en los casos con EC fueron criptitis duodenal en un $26 \%$.

Castellaneta, et al. ${ }^{139}$ realizaron un estudio prospectivo en el que se efectuó una EGD inicial, independientemente de los síntomas del paciente. Se enrolaron 65 pacientes, se eliminaron 11 pacientes que presentaron rectosigmoiditis en la colonoscopia, encontrando anormalidades macroscópicas en el $54 \%$ de los pacientes, el $35 \%$ presentaron granulomas en el tracto gastrointestinal superior, el $20 \%$ fueron diagnosticados por los hallazgos histopatológicos exclusivos de la EGD, encontrando lesiones macroscópicas en el $26 \%$ de los pacientes asintomáticos; por el contrario, en el $33 \%$ de los pacientes sintomáticos no se encontraron anormalidades endoscópicas e histológicas, documentando así la poca correlación clínica y reforzando la utilidad de la EGD. 
Kovacs, et al. ${ }^{140}$ evaluaron el registro nacional de Hungría por tres años y encontraron que la EGD confirmó el diagnóstico de EC como estudio exclusivo en un $9 \%$ y apoyó el diagnóstico de EC en un $36 \%$ de los pacientes que presentaban colitis no clasificada.

Finalmente, se ha documentado una mayor afección gastrointestinal alta en niños que en adultos. Los hallazgos más frecuentemente asociados a Ell son esofagitis linfocítica, gastritis focal, duodenitis y granulomas epitelioides ${ }^{141}$.

\section{Situaciones especiales. En embarazadas} no hay recomendaciones especiales para la endoscopia en enfermedad inflamatoria intestinal. En relación con la sedación en endoscopia en enfermedad inflamatoria intestinal, no hay recomendaciones especiales. Recomendamos revisar el posicionamiento de sedación en endoscopia de la AMEG

GRADE $1 C$ (recomendación fuerte, calidad de evidencia baja).

En la mayoría de las pacientes $(80 \%)$ con Ell que conciben mientras su enfermedad está en remisión, la Ell tiende a permanecer en remisión durante todo el embarazo y en el periodo postparto. De las pacientes con Ell que conciben cuando su enfermedad está activa, el $66 \%$ continúa teniendo enfermedad activa 0 experimentan un empeoramiento de su Ell. Los estudios han demostrado que las tasas de brote de Ell son similares en embarazadas que no embarazadas con Ell. El enfoque diagnóstico para pacientes con Ell que experimentan un brote de la enfermedad durante el embarazo es similar a la evaluación en pacientes no embarazadas. Cuando se realizan procedimientos endoscópicos durante el embarazo, estos deben realizarse de manera preferente durante el segundo trimestre del embarazo ${ }^{142-144}$.

La RSC flexible y la IC con biopsias en las mujeres embarazadas son procedimientos de bajo riesgo. En un estudio que reportó los desenlaces de la RSC en pacientes en todos los trimestres del embarazo se reportó que no hubo complicaciones ni hospitalizaciones asociadas a la realización de procedimientos endoscópicos, y se sugiere que en mujeres que así lo requieran de acuerdo con lo estipulado en esta guía, debe realizarse el estudio y no retrasar el abordaje $^{145}$.

\section{Conclusiones}

La presente guía clínica es el resultado de un análisis multidisciplinario basado en la evidencia disponible hasta el momento de su elaboración y con la opinión de especialistas expertos. Tiene la intención de proveer recomendaciones que ayuden a mejorar y mantener la calidad de la atención de los pacientes con Ell.

\section{Financiamiento}

La presente investigación no ha recibido ninguna beca específica de agencias de los sectores público, comercial, o sin ánimo de lucro.

\section{Conflicto de intereses}

La Dra. Alejandra Noble Lugo es conferencista de Asofarma, Menarini y Takeda.

El Dr. Jony Cerna Cardona es conferencista de Proctor Boston Scientific.

La Dra. Ylse Gutiérrez Grobe es conferencista de Takeda, Abbvie, Janssen y Alfasigma.

El resto de los participantes negaron tener algún conflicto de interés o relación financiera que declarar.

\section{Responsabilidades éticas}

Protección de personas y animales. Los autores declaran que para esta investigación no se han realizado experimentos en seres humanos ni en animales.

Confidencialidad de los datos. Los autores declaran que han seguido los protocolos de su centro de trabajo sobre la publicación de datos de pacientes.

Derecho a la privacidad y consentimiento informado. Los autores declaran que en este artículo no aparecen datos de pacientes.

\section{Bibliografía}

1. Sairenji T, Collins K, Evans D. An update on inflammatory bowel disease. Prim Care Clin Office Pract. 2017;44(4):673-92.

2. Xia B, Crusius J, Meuwissen S, Peña A. Inflammatory bowel disease: definition, epidemiology, etiologic aspects, and immunogenetic studies. World J Gastroenterol. 1998;4(5):446-58.

3. Siegel C, Bernstein C. Identifyng patients with inflamatory bowel diseases at high vs low risk of complications. Clin Gastroenterol Hepatol. 2020;18:1261-7.

4. Jeong D, Kim S, Son M, Son CY, Kim JY, Kronbichler A, et al. Induction and maintenance treatment of inflammatory bowel disease: A comprehensive review. Autoimmun Rev. 2019;18:439-54.

5. Inducing remission in inflammatory bowel disease. Drug Ther Bull. 2003;41:30-2.

6. Nguyen N, Singh S, Sandborn W. Positioning therapies in the management of Crohn's Disease. Clin Gastroenterol Hepatol. 2020;18:1268-79.

7. Danese S, Fiorino G, Peyrin L. Positioning therapies in ulcerative colitis. Clin Gastroenterol Hepatol. 2020;18:1280-90. 
8. Ananthakrishnan AN, Kaplan GG, Ng SC. Changing global epidemiology of inflammatory bowel diseases-Sustaining healthcare delivery into the $21^{\text {st }}$ Century. Clin Gastroenterol Hepatol. 2020;18(6):1252-60.

9. Yan Mak W, Zhao M,Chien S, Burisch J. The epidemiology of inflammatory bowel disease: East meets West. J Gastroenterol Hepatol. 2020;35:380-9.

10. Siew C Ng, Shi H, Hamidi N, Underwood F, Tang W, Benchimol E, et al. Worldwide incidence and prevalence of inflammatory bowel disease in the $21^{\text {st }}$ century: a systematic review of population-based studies. Lancet. 2017;390:2769-78.

11. Calderón $M$, Minckas $N$, Nuñez $S$, Ciapponi A. Inflammatory bowel disease in Latin America: A systematic review. Value Health Reg Issues. 2018;17:126-34

12. Kotze P, Fox E, Cintra A, Geraldo J, Saad-Hossne F, Toro M. Progression of inflammatory bowel diseases throughout Latin America and the Caribbean: A systematic review. Clin Gastroenterol Hepatol. 2020;18: 304-12.

13. Yamamoto-Furusho J. Clinical epidemiology of ulcerative colitis in Mexico. A single hospital-based study in a 20 -year period (1987-2006). J Clin Gastroenterol. 2009;43:221-4.

14. Bosques-Padilla FJ, Sandoval-García ER, MartínezVázquez MA, Garza-González E, Maldonado-Garza HJ. Epidemiología y características clínicas de la colitis ulcerosa crónica idiopática en el noreste de México. Rev Gastroenterol Mex. 2011;76:34-8.

15. Yamamoto-Furusho JK, Bosques-Padilla F, Charúa-Guindic L, Cortés-Espinosa T, Miranda-Cordero R, Saez A, et al. Epidemiología, carga de la enfermedad y tendencias de tratamiento de la enfermedad inflamatoria intestinal en México. Rev Gastroenterol Mex. 2020;85(3):246-56.

16. Yamamoto-Furusho J, Sarmiento-Aguilar A, Toledo-Mauriño J, Bozada-Gutiérrez K, Bosques-Padilla F, Martínez-Vázquez M. Incidence and prevalence of inflammatory bowel disease in Mexico from a nationwide cohort study in a period of 15 years (2000-2017). Medicine. 2019; 8:27-37.

17. Walsh AJ, Ghosh A, Brain AO, Buchel O, Burger D, Thomas S, et al Comparing disease activity indices in ulcerative colitis. $J$ Crohns Colitis. 2014;8:318-25.

18. Truelove SC, Witts LJ. Cortisone in ulcerative colitis. $\mathrm{Br}$ Med J. 1955;2:1041-8

19. Gomollón F, García-López S, Sicilia B, Gisbert JP, Hinojosa J; en representació del Grupo Español de Trabajo de Enfermedad de Crohn y Colitis Ulcerosa or Spanish Group for Working on Crohn's Disease and Ulcerative Colitis (GETECCU). Guía clínica GETECCU del tratamiento de la colitis ulcerosa elaborada con la metodología GRADE. Gastroenterol Hepatol. 2013;36(8):e1-e47.

20. Lobatón T, Bessissow T, De Hertogh G, Lemmens B, Maedler C, van Assche G, et al. The Modified Mayo Endoscopic Score (MMES): a new index for the assessment of extension and severity of endoscopic activity in ulcerative colitis patients. J Crohns Colitis. 2015;9:846-52.

21. Walsh AJ, Bryant RV, Travis SP. Current best practice for disease activity assessment in IBD, Nat Rev Gastroenterol Hepatol. 2016;13:567-79.

22. Lamb CA, Kennedy NA, Raine T, Hendy PA, Smith PJ, Limdi JK, et al. British Society of Gastroenterology consensus guidelines on the management of inflammatory bowel disease in adults. Gut. 2019:68:s1-s106.

23. Lewis JD, Chuai S, Nessel L, Lichtenstein GR, Aberra FN, Ellenberg JH. Use of the noninvasive components of the Mayo score to assess clinical response in ulcerative colitis. Inflamm Bowel Dis. 2008;14:1660-6.

24. Yamamoto-Furusho JK, Bozada-Gutiérrez KE, Sánchez-Rodríguez A, Bojalil-Romano F, Barreto-Zúñiga R, Martínez-Benítez B. Validación de un nuevo índice integral de enfermedad para evaluar el grado de actividad en pacientes mexicanos con colitis ulcerosa: un estudio de cohorte prospectivo. Rev Gastroenterol Mex. 2019;84(3):317-25.

25. Van Hees PA, van Elteren $\mathrm{PH}$, van Lier HJ, van Tongeren JH. An index of inflammatory activity in patients with Crohn's disease. Gut. 1980;21:279-86.

26. Harvey RF, Bradshaw JM. A simple index of Crohn's-disease activity. Lancet. 1980;1:514.

27. Vermeire S, Schreiber S, Sandborn WJ, Dubois C, Rutgeerts P. Correlation between the Crohn's disease activity and Harvey-Bradshaw indices in assessing Crohn's disease severity. Clin Gastroenterol Hepatol. 2010;8:357-63.

28. Peyrin-Biroulet L, Panés J, Sandborn WJ, Vermeire S, Danese S, Feagan BG, et al. Defining disease severity in inflammatory bowel diseases: Current and future directions. Clin Gastroenterol Hepatol. 2016;14:348-54

29. Yamamoto-Furusho JK, Gutiérrez-Grobe Y, López-Gómez JG, Bosques-Padilla F, Rocha-Ramírez JL; Grupo del Consenso Mexicano de Colitis Ulcerosa Crónica Idiopática. Consenso Mexicano para el diagnóstico y tratamiento de la colitis ulcerosa crónica idiopática. Rev Gastroenterol Mex. 2018;83:144-67.

30. Derkacz A, Olczyk P, Komosinska-Vassev K. Diagnostic markers for nonspecific inflammatory bowel diseases. Dis Markers. 2018;2018:7451946.

31. Long MD, Sands BE. What Is the role of the inflammatory bowel disease panel in diagnosis and treatment? Clin Gastroenterol Hepatol. 2018;16:618-20.
32. Capello M, Morreale GC. The role of laboratory tests in Crohn's disease. Clin Med Insights Gastroenterol. 2016;9:51-62.

33. Kiss LS, Papp M, Dorottya B,Vegh Z, Golovics PA, Janka E, et al. High-sensitivity $\mathrm{C}$-reactive protein for identification of disease phenotype, active disease, and clinical relapses in Crohn's disease: A marker for patient classification? Inflamm Bowel Dis. 2012;18:1647-54.

34. Von Roon AC, Karamountzos L, Purkayastha S, Reese GE, Darzi AW, Teare JP, et al. Diagnostic precision of fecal calprotectin for inflammatory bowel disease and colorectal malignancy. Am J Gastroenterol. 2007;102: 803-13.

35. Mosli MH, Zou G, Garg SK, Feagan SG, MacDonald JK, Chande N, et al. C-reactive protein, fecal calprotectin, and stool lactoferrin for detection of endoscopic activity in symptomatic inflammatory bowel disease patients: A systematic review and meta-analysis. Am J Gastroenterol. 2015;110:802-19.

36. Holtman GA, Lisman-van Leeuwen Y, Reitsma JB, Berger MY. Noninvasive tests for inflammatory bowel disease: A meta-analysis. Pediatrics. 2016;137:e20152126.

37. Panés J, Bouhnik Y, Reinisch W, Stoker J, Taylor SA, Baumgart DC, et al. Imaging techniques for assessment of inflammatory bowel disease: Joint ECCO and ESGAR evidence-based consensus guidelines. J Crohns Colitis. 2013;7:556-85

38. Lichtenstein G, Loft E, Isaacs K, Regueiro MD, Gerson LB, Sands BE. ACG clinical guideline: Management of Crohn's disease in adults. Am J Gastroenterol. 2018:113:481-517.

39. Panes J, Bouzas R, Chaparro M, García-Sánchez V, Gisbert JP, Martínez de Guerenu B, et al. Systematic review: the use of ultrasonography, computed tomography and magnetic resonance imaging for the diagnosis, assessment of activity and abdominal complications of Crohn's disease. Aliment Pharmacol Ther. 2011;34:125-45

40. Maaser C, Sturm A, Vavricka S, Kucharzik T, Fiorino G, Annese V, et al. ECCO-ESGAR Guideline for Diagnostic Assessment in IBD Part 1: Initial diagnosis, monitoring of known IBD, detection of complications. J Crohns Colitis. 2019;13-2:144-64.

41. Takenaka K, Ohtsuka K, Kitazume Y, Nagahori M, Fujii T, Saito E, et al. Correlation of the endoscopic and magnetic resonance scoring systems in the deep small intestine in Crohn's disease. Inflamm Bowel Dis. 2015;21:1832-8

42. Rimola J, Rodríguez S, García-Bosch O, Ordas I, Ayala E, Aceituno M, et al. Magnetic resonance for assessment of disease activity and severity in ileocolonic Crohn's disease. Gut. 2009;58:1113-20.

43. American Society for Gastrointestinal Endoscopy Standards of Practice Committee, Shergill AK, Lightdale JR, Bruining DH, Acosta RD, Chandrasekhara V, Chathadi KV, et al. The role of endoscopy in inflammatory bowel disease. Gastrointest Endosc. 2015;81(5):1101-21.

44. Klinger A, Kann B. Endoscopy in inflammatory bowel disease. Surg Clin North Am. 2019;99:1063-82.

45. Flynn S, Eisenstein S. Inflammatory bowel disease presentation and diagnosis. Surg Clin North Am. 2019;99:1051-62.

46. Spiceland CM, Lodhia N. Endoscopy in inflammatory bowel disease: Role in diagnosis, management, and treatment. World $\mathrm{J}$ Gastroenterol. 2018;24(35):4014-20.

47. Dignass A, Eliakim R, Magro F, Maaser C, Chowers $Y$, Geboes K, et al. Second European evidence-based consensus on the diagnosis and management of ulcerative colitis part 1: definitions and diagnosis. J Crohns Colitis. 2012;6:965-90.

48. Mitselos IV, Christodoulou DK, Katsanos KH, Tatsioni A, Rapti A, Eliakim R, et al. The role of small bowel capsule endoscopy and ileocolonoscopy in patients with nonspecific but suggestive symptoms of Crohn's disease. Eur J Gastroenterol Hepatol. 2016;28(8):882-9.

49. Chetcuti S, Ellulb P, Sidhua R. The role of small bowel endoscopy for Crohn's disease. Curr Opin Gastroenterol. 2019;35(3):223-34.

50. Bezzio C, Andreozzi P, Casini V, Manes G, Saibeni S. Patients affected by inflammatory bowel disease: Bowel preparation and sedation. Expert Rev Gastroenterol Hepatol. 2018;12(2):119-24.

51. Tharian B, George N, Navaneethan U. Endoscopy in the diagnosis and management of complications of inflammatory bowel disease. Inflamm Bowel Dis. 2016;22:1184-97.

52. Magro F, Langner C, Driessen A, Ensari A, Geboes K, Mantzaris GJ, et al. European consensus on the histopathology of inflammatory bowel disease. J Crohns Colitis. 2013;7:827-51.

53. Pagnini C, Menasci F, Desideri F, Corleto VD, Delle Fave G, Di Giulio E. Endoscopic scores for inflammatory bowel disease in the era "mucosa healin": Old problem, new perspectives. Dig Liver Dis. 2016;48(7):703-8.

54. Clark C, Turner J. Diagnostic modalites for inflammatory bowel disease. Serologic markers and endoscopy. Surg Clin North Am. 2015;95:1123-41

55. Nguyen Ket S, Palmer R, Travis S. Endoscopic disease activity in inflammatory bowel disease. Curr Gastroenterol Rep. 2015;17:50.

56. Falvey JD, Hoskin T, Meijer B, Ashcroft A, Walmsley R, Day AS, et al Disease activity assessment in IBD: clinical indices and bio-markers fail to predict endoscopic remission. Inflamm Bowel Dis. 2015;21(4):824-31. 
57. Mohammed Vashist N, Samaan M, Mosli MH, Parker CE, MacDonald JK, Nelson SA, et al. Endoscopic scoring indices for evaluation of disease activity in ulcerative colitis. Cochrane Database Syst Rev. 2018;1(1):CD011450

58. Khanna R, Nelson SA, Feagan BG, D'Haens G, Sandborn WJ, Zou GY, et al. Endoscopic scoring indices for evaluation of disease activity in Crohn's disease. Cochrane Database Syst Rev. 2016;2016(8):CD010642.

59. Schoepfer AM, Beglinger C, Straumann A, Trummler M, Vavricka SR, Bruegger LE, et al. Fecal calprotectin correlates more closely with the Simple Endoscopic Score for Crohn's disease (SES-CD) than CRP, blood leukocytes, and the CDAl. Am J Gastroenterol. 2010;105:162-9.

60. Van Assche G. Emerging drugs to treat Crohn's disease. Expert Opin Emerg Drugs. 2007;12:49-59.

61. Rutgeerts P, Geboes K, Vantrappen G, Beyls J, Kerremans R, Hiele M. Predictability of the postoperative course of Crohn's disease. Gastroenterology. 1990;99:956-63.

62. Van Assche G, Dignass A, Reinisch W, van der Woude CJ, Sturm A, de Vos $\mathrm{M}$, et al. The second European evidence-based Consensus on the diagnosis and management of Crohn's disease: Special situations. J Crohns Colitis. 2010;4:63-101.

63. Limdi JK, Picco M, Farraye FA. A review of endoscopic scoring systems and their importance in a treat-to-target approach in inflammatory bowel disease (with videos). Gastrointest Endosc. 2020;91:733-45.

64. Shah SC, Colombel JF, Sands BE, Narula N. Mucosal healing is associated with improved long-term outcomes of patients with ulcerative colitis: a systematic review and meta-analysis. Clin Gastroenterol Hepatol. 2016;14:1245-55

65. Shah SC, Colombel JF, Sands BE, Narula N. Systematic review and meta-analysis: mucosal healing is associated with improved long-term outcomes of patients with Crohn's disease. Aliment Pharmacol Ther. 2016;43:317-33.

66. Peyrin-Biroulet L, Sandborn W, Sands BE, Reinisch W, Bemelman W Bryant RV, et al. Selecting Therapeutic Targets in Inflammatory Bowel Disease (STRIDE): Determining therapeutic goals for treat-to-target. This important study allows evidence based standardisation for targeting remission in IBD. Am J Gastroenterol. 2015;110:1324-38.

67. Zidar N, Ferkolj I, Tepeš K, Stabuck B, Kojc N, Ursic T, et al. Diagnosing cytomegalovirus in patients with inflammatory bowel disease--by immunohistochemistry or polymerase chain reaction? Virchows Arch. 2015;466(5):533-9.

68. Rubin DT, Ananthakrishnan AN, Siegel CA, Sauer BG, Long MD. ACG Clinical Guideline: Ulcerative Colitis in Adults. Am J Gastroenterol. 2019;114(3):384-413

69. Farmer RG, Easley KA, Rankin GB. Clinical patterns, natural history, and progression of ulcerative colitis - A long-term follow-up of 1116 patients. Dig Dis Sci. 1993:38(6):1137-46.

70. Magro F, Gionchetti P, Eliakim R, Ardizzone S, Armuzzi A, Barreiro-de Acosta M, et al. Third European evidence-based consensus on diagnosis and management of ulcerative colitis. Part 1: Definitions, diagnosis, extra-intestinal manifestations, pregnancy, cancer surveillance, surgery, and ileo-anal pouch disorders. J Crohns Colitis. 2017;11(6):649-70.

71. Zenlea T, Yee EU, Rosenberg L, Boyle M, Nanda KS, Wolf JL, et al. Histology grade is independently associated with relapse risk in patients with ulcerative colitis in clinical remission: A prospective study. Am J Gastroenterol. 2016;111(5):685-90.

72. Colombel JF, Ordás I, Ullman T, Rutgeerts P, Chai A, O'Byrne S, et al. Agreement between rectosigmoidoscopy and colonoscopy analyses of disease activity and healing in patients with ulcerative colitis. Gastroenterol. 2016;150(2):389-95.e3.

73. Lin WC, Chang CW, Chen MJ, Hsu TC, Wang HY. Effectiveness of sigmoidoscopy for assessing ulcerative colitis disease activity and therapeutic response. Medicine. 2019;98(21):e15748.

74. Selinger CP, Andrews JM, Titman A, Norton I, Jones DB, McDonald C et al. Long-term follow-up reveals low incidence of colorectal cancer, but frequent need for resection, among Australian patients with inflammatory bowel disease. Clin Gastroenterol Hepatol. 2014:12(4):644-50.

75. Ekbom A, Helmick C, Zack M, Adami HO. Ulcerative colitis and colorectal cancer: A population-based study. N Engl J Med. 1990;323:1228-33

76. Jayaram H, Satsangi J, Chapman RWG. Increased colorectal neoplasia in chronic ulcerative colitis complicated by primary sclerosing cholangitis: Fact or fiction? Gut. 2001;48(3):430-4.

77. Broomé U, Löfberg R, Veress B, Eriksson LS. Primary sclerosing cholangitis and ulcerative colitis: Evidence for increased neoplastic potential. Hepatology. 1995;22(5):1404-8.

78. Lovasz BD, Lakatos L, Golovics PA, David G, Pandur T, Erdelyi Z, et al. Risk of colorectal cancer in Crohn's disease patients with colonic involvement and stenosing disease in a population-based cohort from Hungary. J Gastrointest Liver Dis. 2013;22(3):265-8.

79. Fumery M, Pineton de Chambrun G, Stefanescu C, Buisson A, Bressenot A, Beaugerie L, et al. Detection of dysplasia or cancer in 3.5\% of patients with inflammatory bowel disease and colonic strictures. Clin Gastroenterol Hepatol. 2015;13(10):1770-5.
80. Watanabe T, Aijoka Y, Mitsuyama K, Watanabe K, Hanai H, Nakase H, et al. Comparison of targeted vs random biopsies for surveillance of ulcerative colitis-associated colorectal cancer. Gastroenterology. 2016:151(6):1122-30.

81. Laine L, Kaltenbach T, Barkun A, McQuaid KR, Subramanian V, Soetikno R. SCENIC international consensus statement on surveillance and management of dysplasia in inflammatory bowel disease. Gastroenterology. 2015;148(3):639-651.e28

82. Murthy SK, Kiesslich R. Envolving endoscopic strategies for detection and treatment of neoplastic lesions in inflamatory bowel disease. Gastrointest Endosc. 2013;77:351-9.

83. Kaltenbach T, Sano Y, Friedland S, Soetikno R; American Gastroenterological Association. American Gastroenterological Association (AGA) Institute technology assessment on image enhanced endoscopy. Gastroenterol. 2008:134:327-40.

84. Picco MF, Pasha S, Leighton JA, Bruining D, Loftus Jr EV, Thomas CS, et al. Procedure time and the determination of polypoid abnormalities with experience: implementation of a chromoendoscopy program for surveillance colonoscopy for ulcerative colitis. Inflamm Bowel Dis. 2013;19:1913-20.

85. Flores BM, O'Connor A, Moss AC. Impact of mucosal inflammation on risk of colorectal neoplasia in patients with ulcerative colitis: a systematic review and meta-analysis. Gastrointest Endosc. 2017;86:1006-11.

86. Eaden J, Abrams K, McKay H, Denley H, Mayberry J. Inter-observer variation between general and specialist gastrointestinal pathologists when grading dysplasia in ulcerative colitis. J Pathol. 2001;194(2):152-7.

87. Makkar R, Bo S. Colonoscopic perforation in inflammatory bowel disease. Gastroenterol Hepatol (N Y). 2013:9(9):573-83.

88. Korman LY, Overholt BF, Box T, Winker CK. Perforation during colonoscopy in endoscopic ambulatory surgical centers. Gastrointest Endosc. 2003;58:554-7.

89. Arora G, Mannalithara A, Singh G, Gerson LB, Triadafilopoulos G. Risk of perforation from a colonoscopy in adults: a large population-based study. Gastrointest Endosc. 2009;69(3 Pt 2):654-64.

90. Navaneethan U, Parasa S, Venkatesh PG, Trikudanathan G, Shen B. Prevalence and risk factors for colonic perforation during colonoscopy in hospitalized inflammatory bowel disease patients. J Crohns Colitis. 2011;5(3):189-95

91. Makkar R, Bo S. Colonoscopic perforation in inflammatory bowel disease. Gastroenterol Hepatol (N Y). 2013;9(9):573-83

92. Hong S. Endoscopic therapeutic approach for dysplasia in inflammatory bowel disease. Clin Endosc. 2017:(50):437-45

93. Rubin D, Rothe J, Hetzel J. Are dysplasia and colorectal cancer endoscopically visible in patients with ulcerative colitis? Gastrointest Endosc. 2007;(65): 998-1004

94. Yang DH, Rey I. Endoscopic submucosal dissection for colitis-associated dysplasia. Clin Endosc. 2019;52:120-8.

95. Khalid S, Abbass A, Khetpal N, Shen B, Navaneethan U. Endoscopic detection and resection of dysplasia in inflammatory bowel disease-techniques with videos. Int J Colorectal Dis. 2019;34(4)569-80.

96. Wanders L, Dekker E, Pullens B. Cancer risk after resection of polypoid dysplasia in patients with longstanding ulcerative colitis: a meta-analysis. Clin Gastroenterol Hepatol. 2014:12(5):756-64.

97. Suzuki N, Toyonaga T, East J. Endoscopic submucosal dissection of colitis-related dysplasia. Endoscopy. 2017;49:1237-42.

98. Yang D, Kim J, Song E Chang K Lee SH, Hwang SW, et al Outcomes of ulcerative colitis-associated dysplasia patients referred for potential endoscopic submucosal dissection. J Gastroenterol Hepatol. 2019;34(9):1581-9.

99. Kinoshita S, Uraoka T, Nishizawa T. The role of colorectal endoscopic submucosal dissection in patients with ulcerative colitis. Gastrointestinal Endosc. 2018;87:1079-84.

100. Rieder F, Zimmermann EM, Remzi FH, Sandborn WJ. Crohn's disease complicated by strictures: a systematic review. Gut. 2013:62(7):1072-84

101. Shen B, Kochhar G, Navaneethan U, Farraye FA, Schwartz DA, lacucci M, et al. Practical guidelines on endoscopic treatment for Crohn's disease strictures: a consensus statement from the Global Interventional Inflammatory Bowel Disease Group. Lancet Gastroenterol Hepatol. 2020;5(4):393-405

102. Pokala A, Shen B. Update of endoscopic management of Crohn's disease strictures. Intest Res. 2020:18(1):1-10.

103. Lu C, Holubar SD, Rieder F. How i approach the management of stricturing Crohn's disease. Am J Gastroenterol. 2019;114(8):1181-4.

104. Gu YB, Zhong J; Chinese IBD Endoscopic Club. Endoscopic management of stricturing Crohn's disease. J Dig Dis. 2020;21(6):351-4.

105. Shen B, Kochhar G, Navaneethan U, Liu X, Farraye FA, Gonzalez-Lama Y, et al. Role of interventional inflammatory bowel disease in the era of biologic therapy: a position statement from the Global Interventional IBD Group. Gastrointest Endosc. 2019;89(2):215-37.

106. Bettenworth D, Gustavsson A, Atreja A, Lopez R, Tysk C, van Assche G, et al. A pooled analysis of efficacy, safety, and long-term outcome of endoscopic balloon dilation therapy for patients with stricturing Crohn's disease. Inflamm Bowel Dis. 2017;23(1):133-42. 
107. Bamba S, Sakemi R, Fujii T, Takeda T, Fujioka S, Takenaka K, et al. A nationwide, multi-center, retrospective study of symptomatic small bowe stricture in patients with Crohn's disease. J Gastroenterol. 2020;55(6):615-26.

108. Lan N, Shen B. Multiple primary Crohn's disease-associated strictures had poor response to endoscopic balloon dilation. Am J Gastroenterol. 2018;113:S421-22.

109. Bharadwaj S, Fleshner $P$, Shen B. Therapeutic armamentarium for stricturing Crohn's disease: Medical versus endoscopic versus surgical approaches. Inflamm Bowel Dis. 2015;21(9):2194-213.

110. Shivashankar R, Edakkanambeth Varayil J, Scott Harmsen W, Faubion WA, Wong Kee Song LM, Bruining DH, et al. Outcomes of endoscopic therapy for luminal strictures in Crohn's disease. Inflamm Bowel Dis. 2018;24(7):1575-81.

111. Navaneethan U, Lourdusamy V, Njei B, Shen B. Endoscopic balloon dilation in the management of strictures in Crohn's disease: a systematic review and meta-analysis of non-randomized trials. Surg Endosc. 2016;30(12):5434-43.

112. Morar PS, Faiz O, Warusavitarne J, Brown S, Cohen R, Hind D, et al. Systematic review with meta-analysis: endoscopic balloon dilatation for Crohn's disease strictures. Aliment Pharmacol Ther. 2015;42(10):1137-48.

113. Lan N, Shen B. Endoscopic stricturotomy with needle knife in the treatment of strictures from inflammatory bowel disease. Inflamm Bowel Dis. 2017;23(4):502-13.

114. Lan N, Shen B. Endoscopic stricturotomy versus balloon dilation in the treatment of anastomotic strictures in Crohn's disease. Inflamm Bowel Dis. 2018;24(4):897-907

115. Spinelli A, De Cassan C, Sacchi M, Bazzi P, Danese S, Malesci A, et al Imaging modalities for perianal Crohn's disease. Curr Drug Targets. 2012;13(10):1287-93

116. Terracciano F, Scalisi G, Bossa F, Scimeca D, Biscaglia G, Mangiacotti M, et al. Transperineal ultrasonography: First level exam in IBD patients with perianal disease. Dig Liver Dis. 2016;48(8):874-9.

117. Lee EH, Yang HR, Kim JY. Comparison of transperianal ultrasound with colonoscopy and magnetic resonance imaging in perianal Crohn disease. J Pediatr Gastroenterol Nutr. 2018:66(4):614-9.

118. Wright EK, Novak KL, Lu C, Panaccione R, Ghosh S, Wilson SR. Transperineal ultrasonography in perianal Crohn disease: A valuable imaging modality. Can J Gastroenterol Hepatol. 2015;29(8):445-7.

119. Maconi G, Greco MT, Asthana AK. Transperineal ultrasound for perianal fistulas and abscesses - A systematic review and meta-analysis. Ultraschall Med. 2017;38(3):265-72.

120. Bezzio C, Bryant RV, Manes G, Maconi G, Saibeni S. New horizons in the imaging of perianal Crohn's disease: transperineal ultrasonography. Expert Rev Gastroenterol Hepatol. 2017;11(6):523-30.

121. Lindor KD, Kowdley KV, Harrison ME; American College of Gastroenterology. ACG Clinical Guideline: Primary Sclerosing Cholangitis. Am J Gastroenterol. 2015;110:646-59.

122. Dave M, Elmunzer BJ, Dwamena BA, Higgins PD. Primary sclerosing cholangitis: Meta-analysis of diagnostic performance of MR cholangiopancreatography. Radiology. 2010;256(2):387-96

123. ASGE Standards of Practice Committee, Chandrasekhara V, Khashab MA, Muthusamy VR, Acosta RD, Agrawal D, Bruining DH, et al. Adverse events associated with ERCP. Gastrointest Endosc. 2017;85(1):32-47.

124. Moff SL, Kamel IR, Eustace J, Lawler LP, Kantsevoy S, Kalloo AN, et al. Diagnosis of primary sclerosing cholangitis: a blinded comparative study using magnetic resonance cholangiography and endoscopic retrograde cholangiography. Gastrointest Endosc. 2006;64(2):219-23.

125. Angulo P, Pearce DH, Johnson CD, Henry JJ, LaRusso NF, Petersen BT, et al. Magnetic resonance cholangiography in patients with biliary disease: its role in primary sclerosing cholangitis. J Hepatol. 2000(33):520-7.

126. Chapman MH, Thorburn D, Hirschfield GM, Webster GGJ, Rushbrook SM, Alexander G, et al. British Society of Gastroenterology and UK-PSC guidelines for the diagnosis and managment of primary sclerosing cholangitis. Gut. 2019;(68):1356-78.
127. Björnsson E, Lindqvist-Ottosson J, Asztely M, Olsson R. Dominant strictures in patients with primary sclerosing cholangitis. Am J Gastroenterol. 2004;(99):502-8.

128. Aabakken $\mathrm{L}$, Karlsen $\mathrm{TH}$, Albert $\mathrm{J}$, Arvanitakis $\mathrm{M}$, Chazouilleres $\mathrm{O}$, Dumonceau JM, et al. Role of endoscopy in primary sclerosing cholangitis: European Society of Gastrointestinal Endoscopy (ESGE) and European Association for the Study of the Liver (EASL) Clinical Guideline. J Hepatol. 2017;(66):1265-81.

129. Gotthardt DN, Rudolph G, Kloters-Plachky P, Kulaksiz H, Stiehl A. Endoscopic dilation of dominant stenoses in primary sclerosing cholangitis: outcome after long term treatment. Gastrointest Endosc. 2010:(71):527-34.

130. Jang S, Stevens T, Kou L, Vargo JJ, Parsi MA. Efficacy of digital single-operator cholangioscopy and factors affecting its accuracy in the evaluation of indeterminate biliary stricture. Gastrointest Endosc. 2020;91(2):385-93.

131. Ponsioen CY, Arnelo U, Bergquist A, Rauws EA, Paulsen V, Cantú P, et al. No superiority of stents vs balloon dilatation for dominant strictures in patients with primary sclerosing cholangitis. Gastroenterology. 2018;155:752-9.

132. IBD Working Group pf the ESPGHAN. Inflammatory bowel disease in children and adolescents: Recommendations for diagnosis-The Porto Criteria. J Ped Gastroenterol Nut. 2005;41(1):1-7.

133. Levine A, Koletzko S, Turner D, Escher JC, Cucchiara S, de Ridder L, et al. ESPGHAN revised porto criteria for the diagnosis of inflammatory bowel disease in children and adolescents. J Ped Gastroenterol Nut. 2014;58:795-805

134. Bie CID, Buderus S, Sandhu BK, de Ridder L, Paerregaard A, Veres G, et al. Diagnostic workup of paediatric patients with inflammatory bowel disease in Europe. J Ped Gastroenterol Nut. 2012;54(3):374-80.

135. Lemberg DA, Clarkson CM, Bohane TD, Day AS. Role of esophagogastroduodenoscopy in the initial assessment of children with inflammatory bowel disease. J Gastroenterol Hepatol. 2005;20(11):1696-700.

136. Paerregaard A. What does the IBD patient hide in the upper gastrointestinal tract? Inflam Bowel Dis. 2009;15(7):1101-4.

137. Hummel TZ, Kate FJT, Reitsma JB, Benninga MA, Kindermann A. Additional value of upper $\mathrm{Gl}$ tract endoscopy in the diagnostic assessment of childhood IBD. J Ped Gastroenterol Nut. 2012;54(6):753-7.

138. Tobin JM, Sinha B, Ramani P, Saleh ARH, Murphy MS. Upper gastrointestinal mucosal disease in pediatric Crohn disease and ulcerative colitis: A blinded, controlled study. J Ped Gastroenterol Nut. 2001;32(4):443-8.

139. Castellaneta SP, Afzal NA, Greenberg M, Deere H, Davies S, Murch SH, et al. Diagnostic role of upper gastrointestinal endoscopy in pediatric inflammatory bowel disease. J Ped Gastroenterol Nut. 2004;39(3):257-61.

140. Kovacs M, Muller KE, Arato A, Lakatos PL, Kovacs JB, Varkonyi A, et al. Diagnostic yield of upper endoscopy in paediatric patients with Crohns disease and ulcerative colitis. Subanalysis of the HUPIR registry. J Crohns Colitis. 2012:6(1):86-94.

141. Abuquteish D, Putra J. Upper gastrointestinal tract involvement of pediatric inflammatory bowel disease: A pathological review. World J Gastroenterol. 2019;25(16):1928-35

142. Hashash J, Kane S. Pregnancy and inflammatory bowel disease. Gastroenterol Hepatol. 2015;11(2):96-102.

143. Pedersen N, Bortoli A, Duricova D, Inca R, Panelli MR, Gisbert JP, et al.; European Crohn-Colitis Organisation-ECCO-Study Group of Epidemiology Committee-EpiCom. The course of inflammatory bowel disease during pregnancy and postpartum: a prospective European ECCO-EpiCom Study of 209 pregnant women. Aliment Pharmacol Ther. 2013;38(5): 501-12.

144. ASGE Standard of Practice Committee, Shergill AK, Ben-Menachem T, Chandrasekhara V, Chathadi K, Decker GA, Evans JA, et al. Guidelines for endoscopy in pregnant and lactating women. Gastrointest Endosc. 2012;76(1):18-24

145. Ko MS, Rudrapatna VA, Avila P, Mahadevan U. Safety of flexible sigmoidoscopy in pregnant patients with known or suspect inflammatory bowel disease. Dig Dis Sci. 2020;65(10):2979-85. 